\title{
Effects of Exercise on the Body Composition and Lipid Profile of Individuals with Obesity: A Systematic Review and Meta-Analysis
}

\author{
Kyoung-Bae Kim', Kijin Kim², Changsun Kim³ ${ }^{3}$ Suh-Jung Kang ${ }^{4}$, Hyo Jeong Kim ${ }^{5}$ Seok Yoon ${ }^{6}$, Yun-A Shin ${ }^{7, *}$ \\ ${ }^{1}$ Department of Physical Education, Korea Military Academy, Seoul; ${ }^{2}$ Department of Physical Education, College of Physical Education, Keimyung University, Daegu; \\ ${ }^{3}$ Department of Physical Education, Dongduk Women's University, Seoul: ${ }^{4}$ Department of Sports and Health Management, Sangmyung University, Seoul;: \\ ${ }^{5}$ Department of Sport and Healthy Aging, Korea National Sport University, Seoul, Korea; ${ }^{6}$ Department of Sport Science, Chowan University, Murfreesboro, NC, USA; \\ 'Department of Prescription and Rehabilitation of Exercise, College of Sport Science, Dankook University, Cheonan, Korea
}

Background: Numerous researchers have worked to develop treatments for obesity; however, the prevalence of obesity continues to increase in many countries. Moreover, the effects of physical activity and exercise on obesity remain unclear. Therefore, it is necessary to perform a systematic review and meta-analysis to assess the relationship between exercise and obesity using mediator variables such as the mode of exercise.

Methods: Our review focuses on research tracking the effects of exercise on obesity conducted from 2007 to 2016 and available in any of three databases: Embase, PubMed, and EBSCO Academic Search Premier. The keywords used in the search were "exercise and obesity" and "exercise and obese."

Results: The average size of the effects that exercise interventions have on body mass index (standardized mean difference [SMD], 0.533), waist circumference (SMD, 0.666), total cholesterol (SMD, 0.721), and triglyceride (TG; SMD, 0.603 ) were medium or larger. Exercise had greater effects on the outward appearance of obesity (body mass index, waist circumference) than on its practical factors (weight, \% body fat). The effect of exercise on TG (SMD, 0.603) was larger than that on low-density lipoprotein (SMD, 0.406) and high-density lipoprotein (SMD, -0.222). Exercise duration (weeks of exercise) and intensity correlate better than exercise time (minutes per week) with a large and consistent improvement in adult obesity.

Conclusion: We suggest that individuals with obesity should exercise consistently to achieve significant improvements in their health.

Key words: Obesity, Exercise mode, Body composition, Lipid profile, Meta-analysis
Received May 29, 2019

Reviewed July 10, 2019

Accepted October 30, 2019

${ }^{*}$ Corresponding author

Yun-A Shin

https://orcid.org/0000-0002-8480-3454

Department of Prescription and Rehabilitation of Exercise, College of Sport Science, Dankook University, 119 Dandae-ro, Dongnam-gu, Cheonan 31116, Korea Tel: +82-41-550-3831 Fax: +82-41-550-3831 E-mail: shinagel@dankook.ac.kr

\section{INTRODUCTION}

Obesity is a direct and indirect cause of serious chronic diseases such as hypertension, diabetes, heart disease, and cancer. Many studies during the past few decades have sought solutions to the problem of obesity. Despite those efforts, however, the prevalence of obesity has increased in many countries. In the attempt to fundamentally improve obesity treatment, many new detailed approaches have emerged.
Recent studies have described several interventions for obesity, including diet, physical activity or exercise, behavioral therapy, and medication. Among the various behavioral strategies, exercise interventions can provide effective weight maintenance, weight loss, weight maintenance after loss, and reduction of obesity. However, exercise alone has a limited effect on the body weights of individuals with obesity. ${ }^{1}$ Furthermore, the guidelines for how much physical activity and exercise are needed to improve health are widely known to the public as well as researchers, but they are too general

Copyright (C) 2019 Korean Society for the Study of Obesity

() This is an Open Access article distributed under the terms of the Creative Commons Attribution Non-Commercial License (http://creativecommons.org/licenses/by-nc/4.0/) which permits unrestricted non-commercial use, distribution, and reproduction in any medium, provided the original work is properly cited. 
to specifically address obesity, and the effects are variable and often inconsistent. Thus, it is necessary to understand the magnitude of the effects that physical activity or exercise interventions have on obesity.

Most reviews that have examined the relationship between exercise and obesity have considered only a partial range of factors. For example, the study of Witham and Avenell ${ }^{2}$ included only participants with a mean age $\geq 60$ years. The meta-analysis of Türk et al. ${ }^{3}$ considered only randomized controlled trials (RCTs) that used high intensity exercise with obese patients aged 18 to 60 years. Furthermore, they did not consider the influence of exercise mode (intensity, time, frequency, duration) in their mediation analysis.

Because a systematic review and meta-analysis can statistically integrate multiple independent study variables by their effect sizes for a particular issue and infer overall conclusions, it is a useful strategy in this situation. If we know the average effect size of exercise intensity, time, frequency, and duration in improving obesity, we will be able to concretely establish how different interventions influence the dependent variables, especially weight, body composition, and lipid profile. Therefore, we here include studies of adult subjects to assess the relationship between exercise and obesity using mediator variables. Our purpose in this study was to examine the effectiveness of exercise in terms of time, duration, intensity, and the exercise itself in adults with obesity.

\section{METHODS}

\section{Search strategy and eligibility criteria}

We conducted a literature search of three databases: Embase (http://www.embase.com/), PubMed (http://www.ncbi.nlm.nih. gov/pubmed/), and EBSCO Academic Search Premier (http:// search.ebscohost.com/), to obtain a comprehensive list of studies from 2007 to 2016 that examined the effects of exercise on obesity. The keywords we used were "exercise and obesity" and "exercise and obese." After the initial online search, we selected only RCTs and removed duplicate studies using the EndNote program (Thomson Reuters Co., Toronto, Canada).

At the screening stage, we used the participants, interventions, comparisons, outcomes, timing of outcome measurement, settings, study design (PICOTS-SD) frame to determine the scope of the literature. ${ }^{4}$ Three reviewers (professors with expertise in the area of physical exercise and exercise physiology) screened the titles and abstracts of 4,106 studies based on the inclusion criteria and PICOTS-SD and selected 726 studies. To increase the accuracy of the screening, two other reviewers (professors with expertise in the area of physical exercise and exercise physiology) screened those 726 studies and selected 109 studies. The screening process is summarized in a flowchart (Supplementary Fig. 1): participants: adults with obesity (body mass index [BMI], $\geq 30 \mathrm{~kg} / \mathrm{m}^{2}$; age, 18-65 years); intervention: exercise; comparison: routine care, diet therapy, etc.; outcome: body composition, lipid profile, etc.; time: pre- and posttest studies; study design: RCT.

Full-text articles were then assessed for eligibility. Articles were eliminated if inadequate intervention conditions (e.g., improper control conditions) or insufficient statistical data (e.g., partial or not present) were reported and if weight loss and maintenance for adults with obesity were not the primary focus of the study (e.g., analysis of functional capacity after gastric bypass surgery, breast cancer research). In the end, 64 studies were selected for this systematic review and meta-analysis, and all of them were published in English.

\section{Data extraction and quality assessment}

For the meta-analysis of exercise interventions in adults with obesity, we extracted body composition (weight, BMI, \% body fat, waist circumference) and lipid profile (total cholesterol [TC], triglycerides [TGs], low-density lipoprotein [LDL], and high-density lipoprotein $[\mathrm{HDL}]$ ) data in the form of the mean, standard deviation, and sample sizes for both the treatment and control groups at pre- and post-test. In addition, to analyze the moderator variable effects, we extracted diet control (with or without diet), exercise time (from $<60$ to 450 minutes per week), duration (from $\leq 4$ to 48 weeks), and intensity (low, moderate, vigorous, and high) data about the exercise interventions.

The quality of all studies was assessed using the Physiotherapy Evidence-based Database scale (PEDro) as rated by two authors in this study. The total PEDro score was derived by adding all scale items except for item 1 and specifying the eligibility criteria. ${ }^{4}$ The quality of the studies was assessed using four PEDro score categories: excellent (9-10), good (6-8), fair (4-5), and fail $(\leq 3) .{ }^{5}$ In this study, we considered only studies that scored more than 6 points. 
Disagreements were resolved by discussion.

\section{Reviewer agreement}

Three reviewers (CK, SK, HJK) independently screened the titles and abstracts for eligibility. Agreement among the reviewers was assessed using the kappa statistic. ${ }^{4}$ The three kappa statistics for the

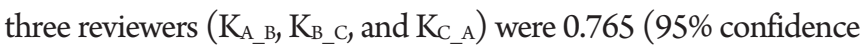
interval [CI], 0.568-0.883), 0.788 (95\% CI, 0.644-0.932), and 0.702 (95\% CI, 0.531-0.873), respectively. After the secondary screening by two other reviewers (KBK, YAS), another kappa statistic $\left(\mathrm{K}_{\mathrm{D}_{-} \mathrm{E}}\right)$ was obtained and determined to be 0.766 (95\% CI, 0.557-0.976). According to Landis \& Koch's guidelines, ${ }^{6}$ all the kappa values indicated substantial agreement, and they were at all acceptable within Fleiss's guidelines.

\section{Statistical analysis}

The meta-analysis and meta-analysis of variance (meta-ANOVA) were performed using $\mathrm{R}$ version 3.5.2 (http://www.r-project.org). The random effects model for computation of the mean effect size was assumed because we considered heterogeneous effect sizes among the studies, including this systematic review and meta-analysis. The moderator variable effects were analyzed by meta-ANO-

VA. Consecutive variables of the moderator were converted into categorical variables for the meta-ANOVA.

\section{Publication bias and heterogeneity}

Publication bias, often called the file-drawer effect because un- published results are imagined to be tucked away in researchers' file cabinets, is a potentially severe impediment to combining the statistical results of studies collected from the literature. To consider publication bias, we used Begg's funnel plots and Egger's regression test with a significance level of 0.10 .

Heterogeneity was tested using $\mathrm{I}^{2}$ values, and the extent of heterogeneity was estimated as follows: low (25\%), moderate (50\%), and high (75\%) $\mathrm{I}^{2}$ values. ${ }^{8}$ For the meta-analysis, the standardized mean difference (SMD) was considered as the mean effect size, and the $95 \% \mathrm{CI}$ and $\mathrm{I}^{2}$ value were obtained using forest plots. The effect sizes were interpreted using Cohen's criteria: small (0.20), medium (0.50), and large (0.80). ${ }^{8}$

\section{RESULTS}

We included 64 articles in this systematic review and meta-analysis study. Twenty-nine articles had only one comparison between the experimental and control groups, and 35 articles had several (2-5) comparisons. Therefore, the total number of cases for comparison was 109. Table 1 presents the characteristics of the studies included in this meta-analysis. ${ }^{9-72}$

\section{Characteristics of the included studies}

The characteristics of the 64 included studies are presented in Table 1. Overall, 5,025 subjects were included in these studies, and the groups ranged in size from 5 to 118 subjects. All studies were published between 2007 and 2017, though the search period rep-

Table 1. The characteristics of the studies included in the systematic review and meta-analysis

\begin{tabular}{|c|c|c|c|c|c|c|c|c|c|}
\hline Author (year) & Group & $\mathrm{N}$ & Age (yr) & Exercise type & $\begin{array}{c}\text { Frequency } \\
\text { (day/wk) }\end{array}$ & $\begin{array}{l}\text { Time } \\
\text { (min) }\end{array}$ & $\begin{array}{l}\text { Duration } \\
\text { (wk) }\end{array}$ & Intensity & $\begin{array}{c}\text { Diet } \\
\text { control }\end{array}$ \\
\hline \multirow{2}{*}{$\begin{array}{l}\text { Herring et al. } \\
(2017)^{9}\end{array}$} & EXP & 12 & $44.3 \pm 7.9$ & \multirow[t]{2}{*}{ Aerobic and resistance training } & \multirow[t]{2}{*}{3} & \multirow[t]{2}{*}{60} & \multirow[t]{2}{*}{12,24} & \multirow[t]{2}{*}{$64 \%-77 \%$ MHR, 60\% 1RM } & \multirow[t]{2}{*}{$\circ$} \\
\hline & $\mathrm{CON}$ & 12 & $52.4 \pm 8.1$ & & & & & & \\
\hline \multirow{3}{*}{$\begin{array}{l}\text { Marcon et al. } \\
(2017)^{10}\end{array}$} & EXP 1 & 22 & $43.4 \pm 2.3$ & Aerobic and stretching exercise & 2 & 25 & 19 & RPE 2-4 & $x$ \\
\hline & EXP2 & 17 & $50.1 \pm 2.8$ & \multirow[t]{2}{*}{ Aerobic and stretching exercise } & \multirow[t]{2}{*}{2} & \multirow[t]{2}{*}{25} & \multirow[t]{2}{*}{19} & \multirow[t]{2}{*}{ RPE 2-4 } & ० \\
\hline & $\mathrm{CON}$ & 18 & $42.5 \pm 2.7$ & & & & & & \\
\hline \multirow{3}{*}{$\begin{array}{l}\text { Zhang et al. } \\
(2017)^{11}\end{array}$} & EXP 1 & 73 & $53.2 \pm 7.1$ & \multirow{3}{*}{$\begin{array}{l}\text { Vigorous/moderate exercise } \\
\text { Moderate exercise }\end{array}$} & & 150/wk & $12 \mathrm{mo}$ & \multirow{3}{*}{$\begin{array}{l}\text { Vigorous/moderate } \\
\text { Moderate }\end{array}$} & $x$ \\
\hline & EXP2 & 73 & $54.4 \pm 7.4$ & & & 150/wk & $12 \mathrm{mo}$ & & $x$ \\
\hline & CON & 74 & $54 \pm 6.8$ & & & & & & $\times$ \\
\hline \multirow{3}{*}{$\begin{array}{l}\text { Ash et al. } \\
(2017)^{12}\end{array}$} & EXP 1 & 6 & $39.7 \pm 4.9$ & Aerobic exercise & 3 & 45 & 8 & $60 \% \mathrm{VO}_{2}$ peak & $x$ \\
\hline & EXP2 & 5 & $43.4 \pm 5.3$ & \multirow[t]{2}{*}{ Isometric handgrip } & 3 & 8 & 8 & $30 \% \mathrm{MVC}$ & $\times$ \\
\hline & CON & 27 & $40.6 \pm 2$ & & & & & & \\
\hline
\end{tabular}


Table 1. Continued

\begin{tabular}{|c|c|c|c|c|c|c|c|c|c|}
\hline Author (year) & Group & $\mathrm{N}$ & Age (yr) & Exercise type & $\begin{array}{l}\text { Frequency } \\
\text { (day/wk) }\end{array}$ & $\begin{array}{l}\text { Time } \\
\text { (min) }\end{array}$ & $\begin{array}{l}\text { Duration } \\
\text { (wk) }\end{array}$ & Intensity & $\begin{array}{c}\text { Diet } \\
\text { control }\end{array}$ \\
\hline \multirow{2}{*}{$\begin{array}{l}\text { Freitas et al. } \\
(2017)^{13}\end{array}$} & EXP & 26 & $45.9 \pm 7.7$ & Aerobic and resistance training & 2 & & $3 \mathrm{mo}$ & $50 \%-75 \% \mathrm{VO}_{2}$ peak & o \\
\hline & $\mathrm{CON}$ & 25 & $48.5 \pm 9.6$ & Stretching and breathing & 2 & & $3 \mathrm{mo}$ & & ० \\
\hline \multirow{2}{*}{$\begin{array}{l}\text { Baillot et al. } \\
(2016)^{14}\end{array}$} & EXP & 8 & 41.4 & Endurance and strength training & 3 & 80 & 12 & & $\circ$ \\
\hline & $\mathrm{CON}$ & 20 & 43.3 & & & & & & o \\
\hline \multirow{3}{*}{$\begin{array}{l}\text { Nikseresht et al. } \\
(2016)^{15}\end{array}$} & EXP 1 & 12 & & Nonlinear resistance training & 3 & 55 & 12 & Very light-very heavy & ० \\
\hline & EXP2 & 10 & & Aerobic interval training & 3 & $4 \min \times 4$ rep & 12 & $90 \%$ HRmax, 65\% HRmax & o \\
\hline & CON & 11 & & & & & & & $\circ$ \\
\hline \multirow{3}{*}{$\begin{array}{l}\text { Nunes et al. } \\
(2016)^{16}\end{array}$} & EXP 1 & 10 & 62 & Low volume resistance training & 3 & & 16 & $70 \% 1 \mathrm{RM} / 3$ sets & $\circ$ \\
\hline & EXP2 & 11 & 62 & High volume resistance training & 3 & & 16 & $70 \% 1 \mathrm{RM} / 6$ sets & $\circ$ \\
\hline & $\mathrm{CON}$ & 11 & 60 & & & & & & \\
\hline \multirow{4}{*}{$\begin{array}{l}\text { Soori et al. } \\
(2017)^{17}\end{array}$} & EXP1 & 8 & & Water-based endurance training & 3 & 45 & 10 & $40 \%-60 \%$ HRmax & $x$ \\
\hline & EXP2 & 8 & & Resistance training & 3 & 10-12 rер & 10 & $40 \%-60 \% 1 \mathrm{RM} / 3$ sets & $\times$ \\
\hline & EXP3 & 8 & & Combined training & 3 & 44 & 10 & $40 \%-60 \%$ HRmax, 40\%-60\% 1RM & $x$ \\
\hline & CON & 8 & & & & & & & $x$ \\
\hline \multirow{2}{*}{$\begin{array}{l}\text { Cuthbertson et al. } \\
(2016)^{18}\end{array}$} & EXP & 30 & 50 & Moderately intense aerobic exercise & $3-5$ & $30-45$ & 16 & $30 \%-60 \%$ HRR & $\times$ \\
\hline & $\mathrm{CON}$ & 20 & 52 & & & & & & $x$ \\
\hline \multirow{4}{*}{$\begin{array}{l}\text { Rafraf et al. } \\
(2015)^{19}\end{array}$} & EXP 1 & 11 & $34.8 \pm 6.2$ & Exercise+carnitine & 3 & & 8 & $55 \%-70 \%$ HRmax & $x$ \\
\hline & EXP2 & 10 & $36.1 \pm 7.2$ & Exercise+placebo & 3 & $30 / 30 \times 3$ & 8 & $55 \%-70 \%$ HRmax & $x$ \\
\hline & CON 1 & 11 & $34.4 \pm 5.5$ & Carnitine & & & & & $x$ \\
\hline & CON 2 & 11 & $36.5 \pm 7.3$ & Placebo & & & & & $x$ \\
\hline \multirow{3}{*}{$\begin{array}{l}\text { Abdelaal and } \\
\text { Mohamad } \\
(2015)^{20}\end{array}$} & EXP 1 & 20 & $52.2 \pm 3$ & Circuit weight training & 3 & & 12 & $60 \%-75 \% 1 \mathrm{RM}$ & $x$ \\
\hline & EXP2 & 20 & $53 \pm 3.5$ & Aerobic exercise training & 3 & $20-35 / 40-50$ & 12 & 60\%-75\% HRmax/RPE 12-14 & $x$ \\
\hline & CON & 19 & $52 \pm 3.3$ & & & & & & \\
\hline \multirow{2}{*}{$\begin{array}{l}\text { Arad et al. } \\
(2015)^{21}\end{array}$} & EXP & 9 & & High intensity interval training & 3 & 24 & 14 & $75 \%-90 \%$ HRR & o \\
\hline & $\mathrm{CON}$ & 11 & & & & & & & $\circ$ \\
\hline \multirow{2}{*}{$\begin{array}{l}\text { Kim et al. } \\
(2015)^{22}\end{array}$} & EXP & 29 & $24.9 \pm 2.8$ & Aerobic exercise & 4 & & 8 & $65 \%-75 \% \mathrm{VO}_{2} \max$ & $\times$ \\
\hline & $\mathrm{CON}$ & 10 & $26.6 \pm 2.8$ & & & & & & $x$ \\
\hline \multirow{4}{*}{$\begin{array}{l}\text { Benito et al. } \\
(2015)^{23}\end{array}$} & EXP 1 & 24 & & Strength training & 3 & 60 & 22 & $50 \%-60 \% 1 \mathrm{RM}$ & ○ \\
\hline & EXP2 & 26 & & Endurance training & 3 & 60 & 22 & $50 \%-60 \%$ HRR & ○ \\
\hline & EXP3 & 24 & & Strength+endurance training & 3 & 60 & 22 & $50 \%-60 \% 1 \mathrm{RM} / 50 \%-60 \%$ HRR & $\circ$ \\
\hline & $\mathrm{CON}$ & 22 & & & & & & & $\circ$ \\
\hline \multirow{2}{*}{$\begin{array}{l}\text { Kordi et al. } \\
(2015)^{24}\end{array}$} & EXP & 14 & $42.2 \pm 14.4$ & Abdominal resistance training & 3 & & 12 & 2 Sets of 8 reps & o \\
\hline & CON & 16 & $43 \pm 15.1$ & & & & & & ० \\
\hline \multirow{2}{*}{$\begin{array}{l}\text { Park et al. } \\
(2015)^{25}\end{array}$} & EXP & 10 & $57.2 \pm 2.6$ & Aerobic+resistance exercise & 4 & $30-40$ & 12 & $40 \%-75 \%$ HRR & $x$ \\
\hline & $\mathrm{CON}$ & 10 & $57.2 \pm 1.7$ & & & & & & \\
\hline \multirow{4}{*}{$\begin{array}{l}\text { Ross et al. } \\
(2015)^{26}\end{array}$} & EXP 1 & 73 & $52.1 \pm 7.4$ & Low amount, low intensity exercise & 5 & $180-300 \mathrm{kcal}$ & 24 & $50 \% \mathrm{VO}_{2}$ peak & $\times$ \\
\hline & EXP2 & 76 & $50.9 \pm 8.6$ & High amount, low intensity exercise & 5 & $300-600 \mathrm{kcal}$ & 24 & $50 \% \mathrm{VO}_{2}$ peak & $\times$ \\
\hline & EXP3 & 76 & $50.3 \pm 8.1$ & High amount, high intensity exercise & 5 & $360-600 \mathrm{kcal}$ & 24 & $75 \% \mathrm{VO}_{2}$ peak & $x$ \\
\hline & $\mathrm{CON}$ & 75 & $52.2 \pm 8.2$ & & & & & & \\
\hline \multirow{2}{*}{$\begin{array}{l}\text { Pugh et al. } \\
(2014)^{27}\end{array}$} & EXP & 34 & 48 & Gymnasium & $3-5$ & $30-45$ & 12 & $45 \%-60 \%$ HRR & $x$ \\
\hline & $\mathrm{CON}$ & 20 & 47 & & & & & & \\
\hline \multirow{3}{*}{$\begin{array}{l}\text { Nikseresht et al. } \\
(2014)^{28}\end{array}$} & EXP 1 & 12 & & Nonlinear resistance training & 3 & $40-65$ & 12 & Very light-very heavy & $x$ \\
\hline & EXP 2 & 12 & & Aerobic interval training & 3 & $16-20$ & 12 & $80 \%-90 \%$ HRmax/3 min recovery & $\times$ \\
\hline & CON & 10 & & & & & & & $x$ \\
\hline \multirow{3}{*}{$\begin{array}{l}\text { Herring et al. } \\
(2014)^{29}\end{array}$} & EXP 1 & 11 & & Resistance exercise & 3 & 60 & 12 & $60 \% 1 \mathrm{RM}$ & $x$ \\
\hline & EXP 2 & 12 & & Aerobic exercise & 3 & 60 & 12 & 50\%-70\% HRR & $x$ \\
\hline & CON & 10 & & & & & & & $\times$ \\
\hline
\end{tabular}

(Continued to the next page) 
Table 1. Continued

\begin{tabular}{|c|c|c|c|c|c|c|c|c|c|}
\hline Author (year) & Group & $\mathrm{N}$ & Age (yr) & Exercise type & $\begin{array}{l}\text { Frequency } \\
\text { (day/wk) }\end{array}$ & $\begin{array}{l}\text { Time } \\
\text { (min) }\end{array}$ & $\begin{array}{l}\text { Duration } \\
\text { (wk) }\end{array}$ & Intensity & $\begin{array}{c}\text { Diet } \\
\text { control }\end{array}$ \\
\hline \multirow{3}{*}{$\begin{array}{l}\text { Irving et al. } \\
(2009)^{30}\end{array}$} & EXP 1 & 13 & $49.2 \pm 1.8$ & Low intensity exercise & 5 & $350-400 \mathrm{kcal}$ & 16 & RPE 10-12 & $x$ \\
\hline & EXP2 & 11 & $49.0 \pm 2.9$ & High intensity exercise & 5 & $350-400 \mathrm{kcal}$ & 16 & 3 Day RPE $15-17 / 2$ day RPE 10-12 & $x$ \\
\hline & CON & 10 & $49.2 \pm 4.8$ & & & & & & $x$ \\
\hline \multirow{3}{*}{$\begin{array}{l}\text { Straznicky et al. } \\
(2010)^{31}\end{array}$} & EXP & 20 & $54 \pm 1$ & Bicycle riding & 3 & 40 & 12 & $65 \% \mathrm{MHR}$ & $x$ \\
\hline & CON 1 & 20 & $55 \pm 1$ & Dietary weight loss & & & & & $\circ$ \\
\hline & CON 2 & 19 & $55 \pm 1$ & No treatment & & & & & \\
\hline \multirow{3}{*}{$\begin{array}{l}\text { Ibáñez et al. } \\
(2010)^{32}\end{array}$} & EXP 1 & 12 & $51.4 \pm 5.5$ & Caloric restriction of $500 \mathrm{kcal} /$ day & 3 & & 16 & & $\circ$ \\
\hline & EXP2 & 13 & & & 2 & $45-60$ & 16 & $50 \%-80 \% 1 \mathrm{RM}$ & $\circ$ \\
\hline & $\mathrm{CON}$ & 9 & & & & & & & $x$ \\
\hline \multirow{3}{*}{$\begin{array}{l}\text { Christiansen et al. } \\
(2010)^{33}\end{array}$} & EXP1 & 25 & & Aerobic exercise & 3 & $60-75$ & 12 & & $x$ \\
\hline & $\mathrm{CON}$ & 29 & & 600 and $800 \mathrm{kcal} /$ day & & & & & $\circ$ \\
\hline & EXP2 & 25 & & 750-1,000 kcal/day, aerobic exercise & 3 & $60-75$ & 12 & & $\circ$ \\
\hline \multirow{2}{*}{$\begin{array}{l}\text { Sartor et al. } \\
(2010)^{34}\end{array}$} & EXP & 10 & $37 \pm 10$ & High intensity interval training & 3 & 40 & 2 & $90 \% \mathrm{VO}_{2}$ peak & $x$ \\
\hline & CON & 9 & $41 \pm 14$ & & & & & & $\times$ \\
\hline \multirow{2}{*}{$\begin{array}{l}\text { Plotnikoff et al. } \\
(2010)^{35}\end{array}$} & EXP & 27 & $55 \pm 12$ & Core and assistance exercise & 3 & & 16 & $80 \% 1 \mathrm{RM}$ & $x$ \\
\hline & $\mathrm{CON}$ & 21 & $54 \pm 12$ & & & & & & $x$ \\
\hline \multirow{2}{*}{$\begin{array}{l}\text { Yamaguchi et al. } \\
(2011)^{36}\end{array}$} & EXP & 11 & $50.0 \pm 3.1$ & Treadmill walking & Every day & 2 rep $\times 30$ min & 4 & Anaerobic threshold & $\circ$ \\
\hline & CON & 8 & $50.0 \pm 2.7$ & Low calorie diet of $25 \mathrm{kcal} / \mathrm{kg}$ & & & 4 & & $\circ$ \\
\hline \multirow{3}{*}{$\begin{array}{l}\text { Straznicky et al. } \\
(2011)^{37}\end{array}$} & EXP & 13 & $52 \pm 1$ & Bicycle riding & 3 & 40 & 12 & $65 \% \mathrm{MHR}$ & $\circ$ \\
\hline & CON 1 & 13 & $55 \pm 2$ & Reduction of 600 calories & & & & & $\circ$ \\
\hline & CON 2 & 12 & $56 \pm 2$ & & & & & & ○ \\
\hline \multirow{3}{*}{$\begin{array}{l}\text { Lim et al. } \\
\qquad(2011)^{38}\end{array}$} & EXP & 99 & $28.0 \pm 0.3$ & Aerobic, resistance exercise & 7 & 60 & 12 & & $\circ$ \\
\hline & CON 1 & 98 & $28.0 \pm 0.3$ & $\begin{array}{l}\text { Metformin, maximum dose of } \\
1,500 \mathrm{mg} / \text { day }\end{array}$ & & & 12 & & \\
\hline & CON 2 & 100 & $28.0 \pm 0.3$ & $\begin{array}{l}\text { Placebo, maximum dose of } \\
1,500 \mathrm{mg} / \text { day }\end{array}$ & & & 12 & & \\
\hline \multirow[t]{3}{*}{$\begin{array}{l}\text { Brinkley et al. } \\
(2011)^{39}\end{array}$} & EXP1 & 15 & $57.3 \pm 5.7$ & $\begin{array}{l}\text { Treadmill 15-20 min at } 45 \%-50 \% \text { of HRR } \\
\text { during first week/hypocaloric menu/ } \\
\text { calcium supplement }\end{array}$ & 3 & 55 & 20 & $45 \%-50 \%$ HRR & ○ \\
\hline & EXP 2 & 8 & $59.4 \pm 4.9$ & $\begin{array}{l}\text { Treadmill 15-20 min at 70\%-75\% of HRR } \\
\text { during first week/hypocaloric menu/ } \\
\text { calcium supplement }\end{array}$ & 3 & 30 & 20 & 70\%-75\% HRR & $\circ$ \\
\hline & CON & 8 & $57.6 \pm 4.8$ & $\begin{array}{l}\text { Hypocaloric menu lunch \& dinner/daily } \\
\text { calcium supplement }(1,000 \mathrm{mg} / \text { day })\end{array}$ & & & 20 & & $\circ$ \\
\hline \multirow{3}{*}{$\begin{array}{l}\text { Brinkley et al. } \\
(2011)^{40}\end{array}$} & EXP 1 & 22 & $58.5 \pm 5.3$ & Treadmill & 3 & $20-55 \mathrm{~min}$ & 20 & $45 \%-50 \%$ HRR & $\circ$ \\
\hline & EXP2 & 17 & $57.2 \pm 4.3$ & Treadmill & 3 & $10-30 \mathrm{~min}$ & 20 & $70 \%-75 \%$ HRR & $\circ$ \\
\hline & CON & 22 & $58.4 \pm 6.2$ & $\begin{array}{l}\text { Hypocaloric menu/allowed } 2 \text { free days } \\
\text { per month/calcium supplement } \\
1,000 \mathrm{mg} / \text { day }\end{array}$ & & & 20 & & ० \\
\hline \multirow{4}{*}{$\begin{array}{l}\text { Atashak et al. } \\
(2011)^{41}\end{array}$} & EXP 1 & 8 & $23.6 \pm 4.4$ & Resistance training protocol & 3 & & 10 & $75 \%-80 \% 1 \mathrm{RM}$ & $\circ$ \\
\hline & EXP2 & 8 & $23.7 \pm 3.8$ & Resistance training protocol & 3 & & 10 & $75 \%-80 \% 1 \mathrm{RM}$ & $\circ$ \\
\hline & CON 1 & 8 & $23.6 \pm 3.3$ & 4 Capsules of ginger rhizome power & $\begin{array}{l}4 \text { times/ } \\
\text { day }\end{array}$ & & 10 & & $\circ$ \\
\hline & CON 2 & 8 & $25.3 \pm 2.2$ & $1 \mathrm{~g}$ of maltodextrin (placebo) & & & 10 & & $x$ \\
\hline \multirow{2}{*}{$\begin{array}{l}\text { Shah et al. } \\
(2011)^{42}\end{array}$} & EXP & 21 & $47.3 \pm 10.0$ & Moderate-intensity aerobic exercise & 5 & & 12 & $60 \%-70 \% \mathrm{VO}_{2} \max$ & $\circ$ \\
\hline & $\mathrm{CON}$ & 12 & $53.9 \pm 8.8$ & & & & & & $\circ$ \\
\hline \multirow{2}{*}{$\begin{array}{l}\text { Castello et al. } \\
(2011)^{43}\end{array}$} & EXP & 11 & $38.4 \pm 4.0$ & Aerobic training on a treadmill & 3 & 61 & 12 & $70 \%$ HRpeak & $x$ \\
\hline & $\mathrm{CON}$ & 10 & $36 \pm 4$ & & & & & & $x$ \\
\hline
\end{tabular}


Table 1. Continued

\begin{tabular}{|c|c|c|c|c|c|c|c|c|c|}
\hline Author (year) & Group & $\mathrm{N}$ & Age (yr) & Exercise type & $\begin{array}{l}\text { Frequency } \\
\text { (day/wk) }\end{array}$ & $\begin{array}{l}\text { Time } \\
\text { (min) }\end{array}$ & $\begin{array}{l}\text { Duration } \\
\text { (wk) }\end{array}$ & Intensity & $\begin{array}{c}\text { Diet } \\
\text { control }\end{array}$ \\
\hline \multirow{3}{*}{$\begin{array}{l}\text { You et al. } \\
(2011)^{44}\end{array}$} & EXP 1 & 13 & $57 \pm 2$ & Treadmill & 3 & 55 & 20 & $45 \%-50 \%$ HRR & o \\
\hline & EXP2 & 8 & $62 \pm 2$ & Treadmill & 3 & 30 & 20 & $70 \%-75 \%$ HRR & $\circ$ \\
\hline & CON & 9 & $59 \pm 2$ & $\begin{array}{l}\text { Hypocaloric menu (RD) \& allowed } \\
2 \text { free day/mo \& calcium supplement } \\
\text { (1,000 mg/day) }\end{array}$ & & & 20 & & $\circ$ \\
\hline \multirow{2}{*}{$\begin{array}{l}\text { Henagan et al. } \\
(2011)^{45}\end{array}$} & EXP & 12 & $65.2 \pm 2.6$ & Progressive RT & 3 & & 12 & 8RM & $\circ$ \\
\hline & $\mathrm{CON}$ & 11 & $66.1 \pm 3.0$ & & & & & & $\circ$ \\
\hline \multirow{2}{*}{$\begin{array}{l}\text { Lee et al. } \\
(2012)^{46}\end{array}$} & EXP & 8 & $54.8 \pm 2.8$ & Yoga exercise & 3 & 60 & 16 & & $x$ \\
\hline & CON & 8 & $54.3 \pm 2.9$ & & & & & & $x$ \\
\hline \multirow{2}{*}{$\begin{array}{l}\text { Sullivan et al. } \\
(2012)^{47}\end{array}$} & EXP & 12 & $48.6 \pm 2.2$ & Walking on a motor-driven treadmill & 5 & $30-60$ & 16 & $45 \%-55 \% \mathrm{VO}_{2} \max$ & $x$ \\
\hline & $\mathrm{CON}$ & 6 & $47.5 \pm 3.1$ & & & & & & $x$ \\
\hline \multirow{4}{*}{$\begin{array}{l}\text { Swift et al. } \\
(2012)^{48}\end{array}$} & EXP 1 & 68 & $57.4 \pm 5.4$ & Cycle ergometer & 3 & 20 & 4 & Pedal cadence of 50 RPM & $x$ \\
\hline & EXP2 & 32 & $55.9 \pm 6.0$ & Cycle ergometer & 3 & 20 & 8 & Pedal cadence of 51 RPM & $x$ \\
\hline & EXP 3 & 32 & $56.3 \pm 6.8$ & Cycle ergometer & 3 & 20 & 12 & Pedal cadence of 52 RPM & $x$ \\
\hline & CON & 23 & $56.8 \pm 5.4$ & & & & & & \\
\hline \multirow{4}{*}{$\begin{array}{l}\text { Foster-Schubert } \\
\text { et al. }(2012)^{49}\end{array}$} & EXP 1 & 117 & $58.1 \pm 5.0$ & Aerobic exercise & 5 & 45 & $12 \mathrm{mo}$ & $70 \%-85 \%$ HRmax & $x$ \\
\hline & EXP2 & 117 & $58.0 \pm 4.5$ & Aerobic exercise \& caloric restriction & 5 & 45 & $12 \mathrm{mo}$ & $70 \%-85 \%$ HRmax & $\circ$ \\
\hline & CON 1 & 87 & $57.4 \pm 4.4$ & & & & & & $x$ \\
\hline & CON 2 & 118 & $58.1 \pm 6.0$ & Caloric deficit of 500-1,000 kcal/day & & & & & $\circ$ \\
\hline \multirow{2}{*}{$\begin{array}{l}\text { Abd El-Kader et al. } \\
(2015)^{50}\end{array}$} & EXP & 39 & $43.6 \pm 6.2$ & Treadmill aerobic exercise & 3 & $10-30$ & $3 \mathrm{mo}$ & $60 \%-70 \%$ HRmax & $x$ \\
\hline & $\mathrm{CON}$ & 39 & $44.1 \pm 5.9$ & No exercise training & & & & & $x$ \\
\hline \multirow[t]{3}{*}{$\begin{array}{l}\text { Wong et al. } \\
(2016)^{51}\end{array}$} & EXP 1 & 14 & $58 \pm 4$ & $\begin{array}{l}\text { WBVT \& intake placebo ( } 4 \text { capsules } \\
\text { before breakfast \& sleeping) }\end{array}$ & 3 & $11-60$ & 8 & $25-40 \mathrm{~Hz}$ & $x$ \\
\hline & EXP2 & 13 & $58 \pm 3$ & WBVT \& intake L-Citrulline & 3 & $11-60$ & 8 & $25-40 \mathrm{~Hz}$ & $\circ$ \\
\hline & $\mathrm{CON}$ & 14 & $58 \pm 4$ & $\begin{array}{l}\text { Intake L-Citrulline ( } 4 \text { capsules before } \\
\text { breakfast \& sleeping) }\end{array}$ & & & & & $\circ$ \\
\hline \multirow{2}{*}{$\begin{array}{l}\text { Osama and } \\
\text { Shehab } \\
(2015)^{52}\end{array}$} & EXP & 50 & $36.4 \pm 5.1$ & Aerobic training & 3 & 40 & 36 & Moderate & $\circ$ \\
\hline & $\mathrm{CON}$ & 50 & $37.2 \pm 4.3$ & & & & 36 & & \\
\hline \multirow{2}{*}{$\begin{array}{l}\text { Franklin et al. } \\
(2015)^{53}\end{array}$} & EXP & 10 & $30.3 \pm 5.4$ & Circuit-based resistance training & 1 & 20 & 8 & 10RM & $x$ \\
\hline & CON & 8 & $30.8 \pm 9.0$ & & & & & & \\
\hline \multirow{2}{*}{$\begin{array}{l}\text { Coen et al. } \\
(2015)^{54}\end{array}$} & EXP & 66 & $41.3 \pm 9.7$ & Semi-supervised moderate exercise & $3-5$ & $147 /$ wk & $6 \mathrm{mo}$ & & $x$ \\
\hline & CON & 62 & $41.9 \pm 10.3$ & Health education & & & & & \\
\hline \multirow{4}{*}{$\begin{array}{l}\text { Romero Moraleda } \\
\text { et al. }(2013)^{55}\end{array}$} & EXP 1 & 24 & $36.1 \pm 8.7$ & Strength training & 3 & $50-60$ & 22 & $50 \%-60 \%$ HRR or $15 R M$ & $\circ$ \\
\hline & EXP2 & 26 & $35.8 \pm 8.0$ & Endurance training & 3 & $50-60$ & 22 & $50 \%-60 \%$ HRR or $15 R M$ & $\circ$ \\
\hline & EXP 3 & 24 & $36.0 \pm 7.3$ & Strength+endurance training & 3 & $50-60$ & 22 & $50 \%-60 \%$ HRR or $15 R M$ & $\circ$ \\
\hline & $\mathrm{CON}$ & 22 & $36.8 \pm 8.9$ & Physical activity & 3 & $50-60$ & 22 & $50 \%-60 \%$ HRR or $15 R M$ & $\circ$ \\
\hline \multirow{3}{*}{$\begin{array}{l}\text { Figueroa et al. } \\
(2013)^{56}\end{array}$} & EXP1 & 13 & $54 \pm 1$ & Low-intensity resistance exercise & 3 & & 12 & & $x$ \\
\hline & EXP2 & 14 & $54 \pm 1$ & Low-intensity resistance exercise & 3 & & 12 & & $\circ$ \\
\hline & $\mathrm{CON}$ & 14 & $55 \pm 1$ & & & & 12 & & $\circ$ \\
\hline \multirow{4}{*}{$\begin{array}{l}\text { Bhutani et al. } \\
(2013)^{57}\end{array}$} & EXP 1 & 18 & $45 \pm 5$ & ADF+exercise & 3 & $35-60$ & 12 & $60 \%-75 \%$ HRmax & $\circ$ \\
\hline & EXP2 & 24 & $42 \pm 2$ & & 3 & $35-60$ & 12 & $60 \%-75 \%$ HRmax & $x$ \\
\hline & CON 1 & 25 & $42 \pm 2$ & Dietary restriction & & & 12 & Controlled feeding & $\circ$ \\
\hline & CON 2 & 16 & $49 \pm 2$ & & & & & & \\
\hline \multirow{3}{*}{$\begin{array}{l}\text { Figueroa et al. } \\
(2013)^{58}\end{array}$} & EXP 1 & 14 & $54 \pm 1$ & Supervised exercise session & $3-4$ & 40 & 12 & 18-22 Repetitions & $x$ \\
\hline & EXP2 & 14 & $54 \pm 1$ & Combination & $3-4$ & 40 & 12 & 18-22 Repetitions & $\circ$ \\
\hline & CON & 13 & $54 \pm 1$ & Commercial weight-loss program & & & 12 & & $\circ$ \\
\hline
\end{tabular}

(Continued to the next page) 
Table 1. Continued

\begin{tabular}{|c|c|c|c|c|c|c|c|c|c|}
\hline Author (year) & Group & $\mathrm{N}$ & Age (yr) & Exercise type & $\begin{array}{l}\text { Frequency } \\
\text { (day/wk) }\end{array}$ & $\begin{array}{l}\text { Time } \\
\text { (min) }\end{array}$ & $\begin{array}{l}\text { Duration } \\
\text { (wk) }\end{array}$ & Intensity & $\begin{array}{l}\text { Diet } \\
\text { control }\end{array}$ \\
\hline \multirow{2}{*}{$\begin{array}{l}\text { Trussardi Fayh } \\
\text { et al. }(2013)^{59}\end{array}$} & EXP & 17 & $32.4 \pm 7$ & Training program & 3 & $30-45$ & 12 & $50 \%-70 \%$ HRR & o \\
\hline & CON & 18 & $30.1 \pm 5.5$ & & & & & & ○ \\
\hline \multirow{3}{*}{$\begin{array}{l}\text { García-Unciti et al. } \\
(2012)^{60}\end{array}$} & EXP & 13 & $48.6 \pm 6.4$ & Resistance training & 2 & & 16 & $50 \%-80 \% 1 \mathrm{RM}$ & $\circ$ \\
\hline & CON 1 & 12 & $51.4 \pm 5.5$ & & & & 16 & & ० \\
\hline & CON 2 & 9 & $50.2 \pm 6.8$ & & & & & & o \\
\hline \multirow[t]{4}{*}{$\begin{array}{l}\text { Tseng et al. } \\
(2013)^{61}\end{array}$} & EXP1 & 10 & $22.2 \pm 0.7$ & 3 Day aerobic, 2 day resistance training & 5 & 60 & 12 & $\begin{array}{l}50 \%-60 \% \text { HRR, } 60 \%-70 \% \text { HRR, } \\
50 \%-60 \% 1 \mathrm{RM}\end{array}$ & ○ \\
\hline & EXP2 & 10 & $22.1 \pm 1.1$ & Aerobic training & 5 & 60 & 12 & $50 \%-60 \%$ HRR, 60\%-70\% HRR & $\circ$ \\
\hline & EXP3 & 10 & $21.3 \pm 0.6$ & Resistance training & 5 & 60 & 12 & $50 \%-60 \% 1 \mathrm{RM}$ & ० \\
\hline & CON & 10 & $22.3 \pm 1.0$ & & & & & & ० \\
\hline \multirow{2}{*}{$\begin{array}{l}\text { Fayh et al. } \\
(2013)^{62}\end{array}$} & EXP & 17 & $32.3 \pm 6.4$ & University gymnasium & 3 & 45 & 65.9 day & $70 \%$ HRR & $\times$ \\
\hline & CON & 18 & $31.4 \pm 5.6$ & Physical activity & & & & & \\
\hline \multirow{2}{*}{$\begin{array}{l}\text { Kim and Kim } \\
(2012)^{63}\end{array}$} & EXP & 15 & $53.5 \pm 2.4$ & Line dance exercise & 3 & 60 & 16 & HRmax-age\% & $\times$ \\
\hline & CON & 15 & $53.5 \pm 2.4$ & & & & & & \\
\hline \multirow{2}{*}{$\begin{array}{l}\text { Snel et al. } \\
(2012)^{64}\end{array}$} & EXP & 13 & $53.0 \pm 2.5$ & Very low calorie diet+exercise & 1 & 30 & 16 & $70 \% \mathrm{VO}_{2} \max$ & $\circ$ \\
\hline & CON & 14 & $56.1 \pm 2.4$ & Very low calorie diet & & & 16 & & ○ \\
\hline \multirow{2}{*}{$\begin{array}{r}\text { Cakmakçi } \\
(2011)^{65}\end{array}$} & EXP & 34 & $36.2 \pm 9.6$ & Pilates training & 4 & 60 & 8 & $60 \%-70 \%$ HRmax & $x$ \\
\hline & CON & 27 & $39.0 \pm 10.0$ & & & & & & \\
\hline \multirow{3}{*}{$\begin{array}{l}\text { Masuo et al. } \\
(2012)^{66}\end{array}$} & EXP 1 & 30 & $37 \pm 6$ & Aerobic exercise or gym exercise & 2 & 60 & 24 & & ० \\
\hline & EXP2 & 30 & $38 \pm 5$ & Aerobic exercise or gym exercise & 2 & 60 & 24 & & $\circ$ \\
\hline & CON & 30 & $38 \pm 5$ & Calorie restriction & & & 24 & & $\circ$ \\
\hline \multirow{6}{*}{$\begin{array}{l}\text { Kerksick et al. } \\
(2010)^{67}\end{array}$} & EXP 1 & 9 & $43 \pm 7$ & Diet+exercise (high energy diet) & 1 & 30 & 14 & $60 \%-80 \%$ HRmax & ○ \\
\hline & EXP2 & 5 & $42 \pm 2$ & No diet+exercise & 2 & 30 & 14 & $60 \%-80 \%$ HRmax & $\times$ \\
\hline & EXP3 & 39 & $38 \pm 8$ & Very low carbohydrate, high protein diet & 1 & 30 & 14 & $60 \%-80 \%$ HRmax & $\circ$ \\
\hline & EXP 4 & 36 & $40 \pm 7$ & Low carbohydrate, moderate protein diet & 1 & 30 & 14 & $60 \%-80 \%$ HRmax & ○ \\
\hline & EXP 5 & 43 & $38 \pm 8$ & High carbohydrate, low protein diet & 1 & 30 & 14 & $60 \%-80 \%$ HRmax & ○ \\
\hline & CON & 9 & $32 \pm 10$ & No diet+no exercise & 2 & & 14 & & $x$ \\
\hline \multirow{4}{*}{$\begin{array}{l}\text { Kadoglou et al. } \\
(2010)^{68}\end{array}$} & EXP1 & 22 & $56.9 \pm 7.1$ & Exercise training & 4 & 60 & $12 \mathrm{mo}$ & $50 \%-80 \% \mathrm{VO}_{2}$ Peak & $\times$ \\
\hline & EXP2 & 23 & $57.8 \pm 7.6$ & Therapy+exercise training & 4 & 60 & $12 \mathrm{mo}$ & $50 \%-80 \% \mathrm{VO}_{2}$ Peak & $x$ \\
\hline & CON & 21 & $60.3 \pm 9.3$ & Maintenance & & & $12 \mathrm{mo}$ & & $\times$ \\
\hline & RSG & 23 & $59.0 \pm 7.4$ & Add -on therapy with rosiglitazone & 7 & & $12 \mathrm{mo}$ & & $x$ \\
\hline \multirow{2}{*}{$\begin{array}{l}\text { Murakami et al. } \\
(2007)^{69}\end{array}$} & EXP & 24 & $51.0 \pm 2.1$ & Diet+exercise & 3 & 60 & 12 & RPM 12-14 & ○ \\
\hline & CON & 18 & $48.2 \pm 1.9$ & Diet & & & 12 & & $\circ$ \\
\hline \multirow[t]{2}{*}{ Arslan $(2011)^{70}$} & EXP & 29 & $41.6 \pm 6.7$ & Step-aerobic dance & 3 & 60 & 8 & $50 \%-85 \% \mathrm{VO}_{2} \max$ & $x$ \\
\hline & CON & 20 & $37.0 \pm 9.1$ & & & & & & $\times$ \\
\hline \multirow{4}{*}{$\begin{array}{l}\text { Mezghanni et al. } \\
(2014)^{71}\end{array}$} & EXP 1 & 11 & $27 \pm 4$ & Moderate intensity training & 5 & 60 & 12 & $50 \% \mathrm{HRR}$ & $x$ \\
\hline & EXP2 & 10 & $25 \pm 5$ & High intensity training & 5 & 60 & 12 & $75 \% \mathrm{HRR}$ & $x$ \\
\hline & EXP 3 & 12 & $28 \pm 5$ & Alternate intensity training & 5 & 60 & 12 & $50 \%-75 \%$ HRR & $\times$ \\
\hline & CON & 10 & $25 \pm 4$ & & & & & & \\
\hline \multirow{2}{*}{$\begin{array}{l}\text { Rshikesan and } \\
\text { Subramanya } \\
(2016)^{72}\end{array}$} & EXP & 37 & $40.0 \pm 8.7$ & Special yoga training (IAYT) & 5 & 90 & 14 & & $x$ \\
\hline & CON & 35 & $42.2 \pm 12.1$ & Regular physical activities & & & & & \\
\hline
\end{tabular}

Values are presented as mean \pm standard deviation.

EXP, experimental group; CON, control group; $\mathrm{MHR}$, maximal heart rate; $\mathrm{RM}$, repetition maximum; $\mathrm{RPE}$, rated perceived exertion; $\mathrm{VO}_{2}$ peak, peak oxygen uptake; $\mathrm{MVC}$, maximum voluntary contraction; rep, repetition; HRmax, maximal heart rate; HRR, heart rate reserve; RD, registered dietitian; RT, resistance training; WBVT, whole-body vibration training; ADF, alternate day fasting; IAYT, integrated approach of yoga therapy. 
resented 10 years (2007-2016).

There were 29 studies with diet control and 35 studies without it. The exercise time per week for the interventions ranged from 20 to 450 minutes, with an average time of 153.3 minutes. The exercise duration varied between 2 and 48 weeks, with an average time of 16.5 weeks. The exercise intensity was classified into four categories (low, moderate, vigorous, and high) using the American College of Sports Medicine (ACSM) Guidelines for Exercise Testing and Prescription. ${ }^{73}$ The ACSM's Foundations of Strength Training and Conditioning classifies the percent 1 repetition maximum (\%1RM) as supramaximal (>100\%), very heavy (95\%-100\%), heavy (90\%95\%), moderately heavy (80\%-90\%), moderate (70\%-80\%), light $(60 \%-70 \%)$ and very light $(<60 \%) .{ }^{74}$ In this study, we used low $(<70 \%)$, moderate $(70 \%-<80 \%)$, vigorous $(80 \%-<90 \%)$, and high ( $\geq 90 \%$ ) (Supplementary Table 1). The PEDro scores of all the included studies varied between 6 and 9 points, with an average of 6.52 points (standard deviation, 0.69 ).

\section{Effects of exercise on body composition and lipid profiles}

We used the SMD as the effect size of each study and present the $95 \% \mathrm{CI}$ of the SMD and $\mathrm{I}^{2}$ value as a measure of heterogeneity in Table 2. Heterogeneity in the effect of exercise on weight in 89 studies was high $\left(\mathrm{I}^{2}=75.9 \%\right)$, with an average SMD of 0.358 in the range from 0.213 to 0.503 . Thus, the average effect size was between small and medium. Heterogeneity in the effect of exercise on BMI in 78 studies was high $\left(\mathrm{I}^{2}=84.8 \%\right)$, with an average SMD of 0.533 in the range from 0.349 to 0.716 . Thus, the average effect size was medium. Heterogeneity in the effect of exercise on \% body fat in 47 studies

Table 2. Effect size of exercise interventions on body composition and lipid profiles

\begin{tabular}{|c|c|c|c|c|c|}
\hline Factor & $\begin{array}{c}\text { Study } \\
\text { (n) }\end{array}$ & $\begin{array}{l}\text { Subject } \\
\text { (n) }\end{array}$ & SMD (95\% CI) & $P$ & $P^{2}(\%)$ \\
\hline Weight (kg) & 89 & 3,665 & 0.358 (0.213 to 0.503$)$ & $<0.001$ & 75.9 \\
\hline BMI $\left(\mathrm{kg} / \mathrm{m}^{2}\right)$ & 78 & 3,682 & 0.533 (0.349 to 0.716$)$ & $<0.001$ & 84.8 \\
\hline Body fat (\%) & 47 & 2,049 & $0.379(0.246$ to 0.512$)$ & $<0.001$ & 44.3 \\
\hline Waist circumference $(\mathrm{cm})$ & 75 & 3,516 & 0.666 (0.478 to 0.854$)$ & $<0.001$ & 84.1 \\
\hline $\mathrm{TC}(\mathrm{mg} / \mathrm{dL})$ & 35 & 906 & 0.721 (0.228 to 1.214 ) & 0.004 & 94.5 \\
\hline $\mathrm{TG}(\mathrm{mg} / \mathrm{dL})$ & 56 & 2,226 & 0.603 (0.257 to 0.949) & $<0.001$ & 92.2 \\
\hline $\mathrm{LDL}(\mathrm{mg} / \mathrm{dL})$ & 49 & 1,994 & 0.406 (0.238 to 0.573$)$ & $<0.001$ & 65.3 \\
\hline $\mathrm{HDL}$ (mg/dL) & 57 & 2,270 & $-0.222(-0.495$ to 0.052$)$ & 0.112 & 88.7 \\
\hline
\end{tabular}

$\mathrm{SMD}$, standardized mean difference; $\mathrm{Cl}$, confidence interval; $\mathrm{BMI}$, body mass index; $\mathrm{TC}$, total cholesterol; TG, triglyceride; LDL, low-density lipoprotein; HDL, high-density lipoprotein. was moderate $\left(\mathrm{I}^{2}=44.3 \%\right)$, with an average SMD of 0.379 in the range from 0.246 to 0.512 . Thus, the average effect size was between small and medium. Heterogeneity in the effect of exercise on waist circumference in 75 studies was high $\left(\mathrm{I}^{2}=84.1 \%\right)$, with an average $\mathrm{SMD}$ of 0.666 in the range from 0.478 to 0.854 . Thus, the average effect size was between medium and large.

Heterogeneity in the effect of exercise on TC in 35 studies was high $\left(\mathrm{I}^{2}=94.5 \%\right)$, with an average SMD of 0.721 in the range from 0.228 to 1.214 , for an almost large average effect size. Heterogeneity in the effect of exercise on TG in 56 studies was high $\left(\mathrm{I}^{2}=92.2 \%\right)$, with an average SMD of 0.603 in the range from 0.257 to 0.949 , for a medium to large average effect size. Heterogeneity in the effect of exercise on LDL in 49 studies was above moderate $\left(\mathrm{I}^{2}=65.3 \%\right)$, with an average $\mathrm{SMD}$ of 0.406 in the range from 0.238 to 0.573 , for a small to medium average effect size. Heterogeneity in the effect of exercise on $\mathrm{HDL}$ in 57 studies was high $\left(\mathrm{I}^{2}=88.7 \%\right)$, with an average $\mathrm{SMD}$ of -0.222 in the range from -0.495 to 0.052 , for a small average effect size.

One result (\% body fat) from the meta-analysis is presented as a forest plot (Fig. 1) because it was the only result that met both the statistical significance and heterogeneity criteria $\left(\mathrm{I}^{2}<50 \%\right)($ Table 2$)$.

With respect to the Egger's regression test for publication bias, the $P$-value was statistically significant for $\%$ body fat $(\mathrm{t}=3.11, \mathrm{df}=45$, $P=0.003)$, waist circumference $(\mathrm{t}=1.95, \mathrm{df}=73, P=0.055)$, and HDL $(\mathrm{t}=-4.58, \mathrm{df}=55, P=0.019)$. The $P$-values for the remaining variables (weight, BMI, TC, TG, and LDL) were not significant. The funnel plot was somewhat asymmetric with respect to \% body fat (Supplementary Fig. 2), though the regression model resulting from Egger's regression test for \% body fat was satisfactory.

\section{Effects of moderators on exercise and obesity}

The meta-ANOVA results showing the effects of the moderators on exercise, obesity, and body composition are shown in Table 3. The average SMD in the studies with diet control was higher than in those with no diet control (except for \% body fat). Regardless of diet, the average SMDs for BMI and waist circumference were close to or above medium (0.452-0.761).

The average SMD for minutes of exercise per week did not yield a specific pattern, possibly because the small number of studies caused inconsistent results. The average SMD for an exercise dura- 


\section{Study}

Herring et al.(2017)

Zhang et al.(2016)

Zhang et al.(2016)_2

Nikseresht et al.(2016)_1

Nikseresht et al.(2016) 2

Soori et al.(2017)_1

Soori et al.(2017)_2

Soori et al.(2017) 3

Cuthbertson et al.(2015)

Arad et al.(2015)

Kim, YS et al.(2015)

Benito et al.(2015)

Benito et al.(2015) 2

Benito et al.(2015)_3

Kordi et al.(2014)

Irving et al.(2009)_ 1

Irving et al.(2009)_2

Plotnikoff et al.(2010)

Brinkley et al.(2011)_1

Brinkley et al.(2011)_2

Atashak et al.(2011)_

Atashak et al.(2011)

Shah et al.(2011)

Castello et al.(2010)

You et al.(2011) _

You et al.(2011) 2

Henagan et al.(2010)

Lee et al.(2012)

Foster-Schubert et al.(2015)

Foster-Schubert et al.(2015)_2

Franklin et al.(2015)

Romero et al.(2013)_1

Romero et al.(2013)_2

Romero et al.(2013) 3

Kim, JW \& DY Kim(2012)

Cakmakci(2010)

Kerksick et al.(2010) 1

Kerksick et al.(2010)_2

Kerksick et al.(2010)_3

Kerksick et al.(2010) 4

Kerksick et al.(2010)_5

Kadoglou et al.(2010)_ 1

Kadoglou et al.(2010) 2

Arslan(2011)

Mezghanni et al.(2014)

Mezghanni et al.(2014) 2

Mezghanni et al.(2014)_3

Random effects mode

Heterogeneity: $I^{2}=44 \%, \tau^{2}=0.0816, p<0.01$

Experimental
Control
Total Mean SD Total Mean SD

$12 \quad 1.005 .22$

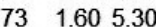

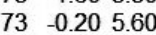

$\begin{array}{lll}12 & 2.30 & 1.85\end{array}$

$10 \quad 2.401 .60$

$\begin{array}{lll}8 & 0.30 & 2.40\end{array}$

$\begin{array}{llll}8 & 0.10 & 1.50\end{array}$

$\begin{array}{llll}8 & 0.50 & 1.90\end{array}$

$\begin{array}{ll}30 & 2.402 .93\end{array}$

$9 \quad 0.903 .85$

293.963 .81

$24 \quad 5.246 .10$

$26 \quad 5.346 .13$

$\begin{array}{ll}24 & 4.85 \\ 6.94\end{array}$

$\begin{array}{lll}14 & 2.20 & 3.87\end{array}$

$\begin{array}{llll}13 & 0.70 & 1.60\end{array}$

$\begin{array}{lll}11 & 1.60 & 1.70\end{array}$

$\begin{array}{llll}27 & -0.10 & 6.75\end{array}$

$\begin{array}{llll}15 & 4.30 & 2.77\end{array}$

$\begin{array}{llll}9 & 3.902 .83\end{array}$

$8 \quad 3.103 .40$

$8 \quad 5.002 .61$

$\begin{array}{lll}21 & 0.90 & 4.98\end{array}$

$\begin{array}{lll}11 & 8.00 & 1.30\end{array}$

$\begin{array}{llll}13 & 4.10 & 0.90\end{array}$

$\begin{array}{llll}8 & 5.80 & 1.02\end{array}$

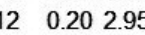

$\begin{array}{lll}8 & 2313.18\end{array}$

$117 \quad 1.604 .52$

$117 \quad 5.905 .88$

$\begin{array}{lll}10 & 1.00 & 4.25\end{array}$

$24 \quad 5.306 .22$

$\begin{array}{lll}26 & 5.10 & 6.01\end{array}$

$\begin{array}{lll}24 & 4.60 & 7.34\end{array}$

$\begin{array}{lll}15 & 2.49 & 3.14\end{array}$

$\begin{array}{lll}34 & 2.39 & 3.20\end{array}$

$\begin{array}{ll}9 & 0.304 .45\end{array}$

$\begin{array}{ll}5 & 2.004 .60\end{array}$

$\begin{array}{lll}39 & 2.20 & 4.72\end{array}$

$\begin{array}{ll}36 & 1.904 .60\end{array}$

$\begin{array}{llll}43 & 1.60 & 4.11\end{array}$

$\begin{array}{ll}22 & 1.605 .32\end{array}$

$\begin{array}{ll}23 & 2.405 .85\end{array}$

$\begin{array}{lll}29 & 1.76 & 2.57\end{array}$

$\begin{array}{llll}11 & 2.80 & 2.46\end{array}$

$\begin{array}{llll}10 & 3.70 & 2.77\end{array}$

$\begin{array}{ll}12 \quad 3.20 & 2.94\end{array}$

130

$\begin{array}{lll}74 & -0.30 & 4.29\end{array}$

$\begin{array}{lll}74 & 0.30 & 7.05\end{array}$

$\begin{array}{lll}11 & -0.40 & 1.47\end{array}$

$\begin{array}{llll}11 & -0.40 & 1.47\end{array}$

$\begin{array}{llll}8 & 0.00 & 1.15\end{array}$

$8 \quad 0.001 .15$

$\begin{array}{lll}20 & 0.30 & 5.60\end{array}$

$\begin{array}{lll}11 & 0.906 .67\end{array}$

$\begin{array}{llll}10 & -0.07 & 2.70\end{array}$

$\begin{array}{lll}22 & 4.89 & 6.20\end{array}$

$22 \quad 4.896 .20$

$\begin{array}{lll}22 & 4.89 & 6.20\end{array}$

$\begin{array}{lll}16 & 1.70 & 4.25\end{array}$

$\begin{array}{lll}10 & 1.20 & 2.08\end{array}$

$\begin{array}{lll}10 & 1.20 & 2.08\end{array}$

$\begin{array}{lll}8 & 3.30 & 3.10\end{array}$

$8 \quad 3.303 .10$

$8 \quad 0.102 .85$

$\begin{array}{llll}8 & 0.40 & 2.69\end{array}$

$\begin{array}{lll}12 & 1.70 & 4.59\end{array}$

$\begin{array}{lll}10 & 6.00 & 1.32\end{array}$

$\begin{array}{llll}9 & 3.10 & 1.43\end{array}$

$\begin{array}{llll}9 & 3.10 & 1.43\end{array}$

110.502 .95

$\begin{array}{llll}8 & -0.80 & 2.32\end{array}$

$\begin{array}{lll}87 & 0.20 & 4.82\end{array}$

$\begin{array}{ll}118 \quad 4.20 & 5.57\end{array}$

$\begin{array}{llll}8 & -0.70 & 7.61\end{array}$

$\begin{array}{lll}22 & 4.90 & 6.17\end{array}$

$\begin{array}{lll}22 & 4.90 & 6.17\end{array}$

$\begin{array}{lll}22 & 4.90 & 6.17\end{array}$

$\begin{array}{lll}15 & -0.94 & 2.04\end{array}$

$\begin{array}{lll}27 & -0.06 & 3.19\end{array}$

$\begin{array}{lll}9 & 1.00 & 2.41\end{array}$

$\begin{array}{lll}9 & 1.002 .41\end{array}$

$\begin{array}{lll}9 & 1.002 .41\end{array}$

$\begin{array}{lll}9 & 1.002 .41\end{array}$

$\begin{array}{lll}9 & 1.002 .41\end{array}$

$21 \quad 0.406 .65$

$\begin{array}{llll}20 & -0.05 & 3.41\end{array}$

$\begin{array}{llll}10 & -0.10 & 2.04\end{array}$

$\begin{array}{llll}10 & -0.10 & 2.04\end{array}$

$\begin{array}{lll}10 & -0.10 & 2.04\end{array}$

919 $\begin{array}{lll}8 & 0.00 & 1.15\end{array}$

$21 \quad 0.107 .30$

$\begin{array}{ll}21 & 0.406 .65\end{array}$

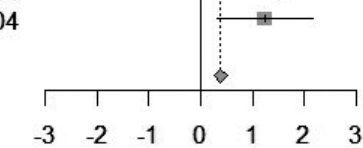

SMD

95\%-Cl Weight

Difference

$0.26[-0.54 ; 1.07] \quad 1.8 \%$

$0.21 \quad[-0.12 ; 0.53] \quad 4.2 \%$

$-0.08[-0.40 ; 0.25] \quad 4.2 \%$

$1.55[0.59 ; 2.50] \quad 1.4 \%$

$1.75[0.71 ; 2.79] \quad 1.3 \%$

$0.15[-0.83 ; 1.13] \quad 1.4 \%$

$0.07 \quad[-0.91 ; 1.05] \quad 1.4 \%$

$0.30[-0.69 ; 1.29] \quad 1.4 \%$

$0.49[-0.08 ; 1.07] \quad 2.8 \%$

$0.00[-0.88 ; 0.88] \quad 1.6 \%$

$1.11[0.34 ; 1.87] \quad 2.0 \%$

$0.06[-0.52 ; 0.63] \quad 2.7 \%$

$0.07[-0.50 ; 0.64] \quad 2.8 \%$

$-0.01 \quad[-0.58 ; 0.57] \quad 2.7 \%$

$0.12[-0.60 ; 0.84] \quad 2.1 \%$

$-0.26[-1.09 ; 0.56] \quad 1.8 \%$

$0.20[-0.66 ; 1.06] \quad 1.7 \%$

$-0.03[-0.60 ; 0.54] \quad 2.8 \%$

$0.33[-0.53 \cdot 120] \quad 1.7 \%$

$0.19[-0.76 ; 1.15] \quad 1.4 \%$

$0.90[-0.14 ; 1.95] \quad 1.3 \%$

$1.64[0.46 ; 2.82] \quad 1.0 \%$

$-0.16[-0.87 ; 0.55] \quad 2.2 \%$

$1.47[0.48 ; 2.45] \quad 1.4 \%$

$0.84[-0.05 ; 1.74] \quad 1.6 \%$

$2.04[0.81 ; 3.27] \quad 1.0 \%$

$-0.10[-0.92 ; 0.72] \quad 1.8 \%$

$1.06[-0.01 ; 2.12] \quad 1.2 \%$

$0.30 \quad[0.02 ; 0.58] \quad 4.5 \%$

$0.30[0.04 ; 0.55] \quad 4.7 \%$

$0.27[-0.66 ; 1.21] \quad 1.5 \%$

$0.06[-0.52 ; 0.64] \quad 2.7 \%$

$0.03[-0.54 ; 0.60] \quad 2.8 \%$

$-0.04 \quad[-0.62 ; 0.54] \quad 2.7 \%$

$1.26[0.47 ; 2.05] \quad 1.9 \%$

$0.76[0.23 ; 1.28] \quad 3.0 \%$

$-0.19[-1.11 ; 0.74] \quad 1.5 \%$

$0.28[-0.82 ; 1.38] \quad 1.2 \%$

$0.27[-0.46 ; 0.99] \quad 2.1 \%$

$0.21[-0.53: 0.94] \quad 21 \%$

$0.15[-0.57 ; 0.87] \quad 2.1 \%$

$0.20[-0.40 ; 0.80] \quad 2.6 \%$

$0.31 \quad[-0.28 ; 0.91] \quad 2.7 \%$

$0.61 \quad[0.02 ; 1.19] \quad 2.7 \%$

$1.23[0.28 ; 2.18] \quad 1.5 \%$

$1.50[0.48 ; 2.51] \quad 1.3 \%$

$1.23[0.30 ; 2.16] \quad 1.5 \%$

$0.38[0.25 ; 0.51] 100.0 \%$

Figure 1. Forest plot of $\%$ body fat. The meta-analysis result of $\%$ body fat is presented because only \% body fat met both the statistical significance and heterogeneity criteria $\left(I^{2}<50 \%\right)$. "Total" in the figure indicates the number of subjects. Thirteen articles had several ( $2-5$ pieces) comparisons, and each comparison was presented independently in the plot. SD, standard deviation; SMD, standardized mean difference; $\mathrm{Cl}$, confidence interval.

tion of 12 weeks was close to or above medium for all 4 variables (weight, BMI, \% body fat, waist circumference). This was especially the case for BMI (0.911) and waist circumference (0.910), which had large average SMDs.

The average SMD for exercise intensity was between 0.043 (\% body fat) and 0.609 (BMI) for the low intensity case, 0.346 (weight) and 0.874 (waist circumference) for the moderate intensity case, and 0.293 (weight) and 0.613 (waist circumference) for the vigorous intensity case; no studies used high intensity exercise. The av- erage SMD for the moderate intensity case was higher than that for the low and vigorous cases for BMI, \% body fat, and waist circumference. Only with respect to weight was the average SMD higher at low intensity.

The meta-ANOVA results for the effects of the moderators on exercise, obesity, and lipid profile are shown in Table 4. The average SMD for diet treatment was above medium for TC (0.825) and TG (0.772). The average SMD for HDL was smaller than that for the other factors. The average SMD for exercise time per week was 
Table 3. Meta-ANOVA results for moderator effects on body composition

\begin{tabular}{|c|c|c|c|c|c|c|c|c|}
\hline \multirow{2}{*}{ Moderator } & \multicolumn{2}{|l|}{ Weight } & \multicolumn{2}{|l|}{$\mathrm{BMl}$} & \multicolumn{2}{|l|}{$\%$ Body fat } & \multicolumn{2}{|l|}{ Waist circumference } \\
\hline & SMD (95\% Cl) & $n$ & SMD (95\% CI) & $n$ & $\mathrm{SMD}(95 \% \mathrm{Cl})$ & $n$ & $\mathrm{SMD}(95 \% \mathrm{Cl})$ & $\mathrm{n}$ \\
\hline \multicolumn{9}{|l|}{ Diet control } \\
\hline With diet & 0.433 (0.205 to 0.661) & 47 & 0.608 (0.294 to 0.922) & 38 & 0.296 (0.106 to 0.485) & 204 & 0.761 (0.465 to 1.056$)$ & 39 \\
\hline No diet & 0.255 (0.099 to 0.412) & 42 & 0.452 (0.257 to 0.648) & 40 & 0.464 (0.273 to 0.655$)$ & 23 & 0.562 (0.333 to 0.792) & 36 \\
\hline \multicolumn{9}{|c|}{ Exercise time (min/wk) } \\
\hline$<60$ & 0.899 (0.335 to 1.463$)$ & 5 & $0.075(-0.855$ to 1.005$)$ & 1 & $0.995(-0.457$ to 2.446$)$ & 2 & 0.771 (0.242 to 1.300) & 3 \\
\hline $60-89$ & 0.002 (-0.255 to 0.258) & 5 & 0.360 (-0.433 to 1.153$)$ & 3 & $0.000(-0.881$ to 0.881$)$ & 1 & $0.089(-0.179$ to 0.358$)$ & 4 \\
\hline 90-119 & $0.154(-0.081$ to 0.389$)$ & 10 & 0.391 (0.006 to 0.777) & 9 & $0.193(-0.763$ to 1.148$)$ & 1 & 0.315 (0.034 to 0.595) & 7 \\
\hline $120-149$ & 0.373 (0.091 to 0.656) & 17 & 0.615 (0.283 to 0.947) & 14 & 0.293 (0.110 to 0.475$)$ & 4 & 0.976 (0.472 to 1.480$)$ & 9 \\
\hline $150-179$ & 0.052 (-0.194 to 0.298$)$ & 7 & 0.243 (0.075 to 0.411) & 8 & $0.200(-0.051$ to 0.450$)$ & 8 & 0.476 (0.234 to 0.718$)$ & 7 \\
\hline 180-209 & $0.208(-0.045$ to 0.460$)$ & 13 & $0.257(-0.003$ to 0.517$)$ & 13 & 0.498 (0.125 to 0.871) & 8 & $0.362(-0.027$ to 0.750$)$ & 13 \\
\hline 210-239 & $0.092(-0.889$ to 1.072$)$ & 1 & 0.063 (-0.917 to 1.043$)$ & 1 & - & - & - & - \\
\hline 240 & $0.303(-0.206$ to 0.811$)$ & 1 & 0.283 (-0.042 to 0.608) & 3 & 0.450 (0.104 to 0.796) & 3 & $0.331(-0.178$ to 0.840$)$ & 1 \\
\hline 300 & 1.237 (0.464 to 2.009) & 6 & 1.643 (0.795 to 2.490) & 6 & 1.309 (0.753 to 1.866$)$ & 3 & 2.290 (1.311 to 3.268) & 6 \\
\hline $420-450$ & $0.078(-0.334$ to 0.491$)$ & 2 & $0.103(-0.310$ to 0.515$)$ & 2 & - & - & $-0.288(-0.752$ to 0.177$)$ & 1 \\
\hline \multicolumn{9}{|l|}{ Duration (wk) } \\
\hline$\leq 4$ & $-0.028(-0.408$ to 0.353$)$ & 3 & 0.017 (-0.893 to 0.928) & 1 & - & - & $0.087(-0.386$ to 0.560$)$ & 1 \\
\hline 8-11 & $0.237(0.032$ to 0.441$)$ & 12 & 0.322 (0.104 to 0.540) & 13 & 0.655 (0.390 to 0.921) & 9 & 0.706 (0.226 to 1.186) & 7 \\
\hline $12-15$ & 0.455 (0.179 to 0.731$)$ & 43 & 0.911 (0.501 to 1.322 ) & 33 & 0.541 (0.216 to 0.866) & 16 & 0.910 (0.573 to 1.246$)$ & 41 \\
\hline $16-19$ & 0.576 (0.266 to 0.886$)$ & 13 & 0.431 (0.169 to 0.694) & 10 & $0.411(-0.041$ to 0.864$)$ & 6 & 0.600 (0.242 to 0.959$)$ & 11 \\
\hline $20-23$ & $0.114(-0.085$ to 0.313$)$ & 11 & 0.150 (-0.047 to 0.348) & 10 & $0.235(-0.052$ to 0.522$)$ & 9 & $0.143(-0.085$ to 0.372$)$ & 9 \\
\hline 24 & $0.120(-0.499$ to 0.738$)$ & 3 & $0.096(-0.556$ to 0.748$)$ & 3 & - & - & $0.117(-0.230$ to 0.464$)$ & 1 \\
\hline 36 & - & - & 0.642 (0.240 to 1.045) & 1 & - & - & - & - \\
\hline 48 & $0.129(-0.059$ to 0.318$)$ & 2 & 0.214 (0.078 to 0.352) & 6 & 0.209 (0.071 to 0.347) & 6 & 0.284 (0.138 to 0.430) & 4 \\
\hline \multicolumn{9}{|l|}{ Intensity } \\
\hline Low & 0.533 (0.022 to 1.044) & 11 & 0.609 (0.079 to 1.140) & 7 & $0.043(-0.322$ to 0.407$)$ & 5 & $0.558(-0.446$ to 1.562$)$ & 4 \\
\hline Moderate & 0.346 (0.165 to 0.526$)$ & 28 & 0.627 (0.386 to 0.868) & 31 & 0.457 (0.181 to 0.733) & 16 & 0.874 (0.566 to 1.182) & 27 \\
\hline Vigorous & 0.293 (0.136 to 0.450$)$ & 28 & 0.370 (0.184 to 0.557$)$ & 23 & 0.345 (0.189 to 0.501$)$ & 21 & 0.613 (0.387 to 0.838) & 27 \\
\hline
\end{tabular}

Meta-ANOVA, meta-analysis of variance; BMI, body mass index; SMD, standardized mean difference; $\mathrm{Cl}$, confidence interval; n, number of studies.

above medium and ranged from -0.506 (HDL) to 0.709 (TG) for 120 to 149 minutes of exercise per week. Similar to the results for body composition, the average SMD for exercise time per week did not appear to follow a specific pattern as exercise time increased. For this attribute also, the number of studies was small, which might have caused inconsistent results.

The average SMD for the effect of exercise duration on TC and TG increased rapidly from 12 weeks. The average SMD at 36 weeks was very large and ranged from -0.993 (HDL) to 2.585 (TG).

The average SMD for exercise intensity was between -1.675 (HDL) and 1.191 (TG) for the low intensity case, -0.023 (HDL) and 0.506 (LDL) for the moderate intensity case, and -0.285 (HDL) and 0.410 (TG) for the vigorous intensity case. The average SMD for the low intensity case was far greater than that of the other cases.
The meta-ANOVA results for one variable ( $\%$ body fat) are presented in the form of a forest plot (Fig. 2).

\section{DISCUSSION}

For this study, we performed a systematic review and meta-ANOVA to examine the effect of exercise on both the body composition and lipid profiles of adults with obesity. According to Table 2, the average effect size of exercise on BMI (SMD, 0.533), waist circumference (SMD, 0.666), TC (SMD, 0.721), and TG (SMD, 0.603) was above medium. The average effect size on weight (SMD, 0.358), $\%$ body fat (SMD, 0.379), LDL (SMD, 0.406), and HDL (SMD, $-0.222)$ was below medium. The heterogeneity of most of the dependent variables was high $\left(\mathrm{I}^{2}=75.9 \%-94.5 \%\right)$, except for $\mathrm{LDL}$ 
Table 4. Meta-ANOVA results for the effect of exercise on lipid profile variables

\begin{tabular}{|c|c|c|c|c|c|c|c|c|}
\hline \multirow{2}{*}{ Moderator } & \multicolumn{2}{|l|}{ TC } & \multicolumn{2}{|l|}{ TG } & \multicolumn{2}{|l|}{$\mathrm{LDL}$} & \multicolumn{2}{|l|}{ HDL } \\
\hline & SMD $(95 \%$ Cl) & $n$ & SMD $(95 \%$ CI) & $\mathrm{n}$ & $\mathrm{SMD}(95 \% \mathrm{Cl})$ & $n$ & SMD $(95 \%$ Cl) & $n$ \\
\hline \multicolumn{9}{|l|}{ Diet control } \\
\hline With diet & 0.825 (0.050 to 1.601) & 22 & 0.772 (0.184 to 1.359) & 25 & 0.340 (0.092 to 0.588) & 27 & $-0.116(-0.549$ to 0.317$)$ & 29 \\
\hline No diet & 0.449 (0.172 to 0.727$)$ & 13 & 0.377 (0.114 to 0.641) & 31 & 0.468 (0.259 to 0.677) & 22 & $-0.318(-0.611$ to -0.024$)$ & 28 \\
\hline \multicolumn{9}{|c|}{ Time (min/wk) } \\
\hline$<60$ & $-0.019(-0.949$ to 0.911$)$ & 1 & $1.509(-0.031$ to 3.049$)$ & 3 & $0.232(-0.404$ to 0.869$)$ & 2 & -2.441 (-4.439 to -0.443$)$ & 4 \\
\hline $60-89$ & $-0.044(-0.677$ to 0.589$)$ & 1 & $-0.281(-0.918$ to 0.355$)$ & 1 & $-0.076(-0.709$ to 0.558$)$ & 1 & $0.220(-0.415$ to 0.855$)$ & 1 \\
\hline 90-119 & 0.421 (0.023 to 0.819) & 5 & $-0.131(-0.468$ to 0.206$)$ & 5 & 0.274 (-0.081 to 0.628) & 5 & 0.443 (0.100 to 0.786) & 5 \\
\hline $120-149$ & 0.656 (0.028 to 1.284) & 6 & 0.709 (0.109 to 1.308) & 12 & 0.578 (0.100 to 1.056) & 10 & $-0.506(-1.075$ to 0.063$)$ & 12 \\
\hline $150-179$ & $0.040(-0.215$ to 0.294$)$ & 5 & $-0.195(-0.550$ to 0.159$)$ & 6 & $0.028(-0.215$ to 0.271$)$ & 6 & $-0.179(-0.436$ to 0.080$)$ & 6 \\
\hline 180-209 & 1.010 (0.641 to 1.558) & 3 & $0.193(-0.384$ to 0.769$)$ & 4 & 0.527 (0.096 to 0.958) & 3 & $-0.359(-0.667$ to -0.050$)$ & 5 \\
\hline $210-239$ & 1.733 (0.562 to 2.904) & 1 & $0.141(-0.841$ to 1.122$)$ & 1 & $0.406(-0.586$ to 1.397$)$ & 1 & 0.495 (0.502 to 1.492) & 1 \\
\hline 240 & 0.575 (0.144 to 1.005$)$ & 2 & 0.634 (0.202 to 1.066) & 2 & 1.164 (0.706 to 1.623) & 2 & $-1.020(-1.631$ to -0.409$)$ & 2 \\
\hline 300 & - & - & 0.904 (0.040 to 1.769) & 6 & 0.876 (0.350 to 1.401) & 3 & $0.173(-0.324$ to 0.669$)$ & 3 \\
\hline $420-450$ & $-0.585(-1.519$ to 0.350$)$ & 1 & $-0.016(-0.927$ to 0.895$)$ & 1 & $-0.548(-1.480$ to 0.383$)$ & 1 & $0.505(-0.423$ to 1.434$)$ & 1 \\
\hline \multicolumn{9}{|c|}{ Duration (wk) } \\
\hline$\leq 4$ & $-0.585(-1.519$ to 0.350$)$ & 1 & 0.012 (-0.628 to 0.653) & 2 & $-0.301(-0.949$ to 0.346$)$ & 2 & $0.083(-0.734$ to 0.899$)$ & 2 \\
\hline 8-11 & $0.332(-0.235$ to 0.898$)$ & 3 & $0.274(-0.105$ to 0.652$)$ & 6 & 0.484 (0.105 to 0.862) & 7 & $-0.452(-0.807$ to -0.100$)$ & 7 \\
\hline $12-15$ & $1.165(-0.116$ to 2.447$)$ & 13 & 0.834 (0.161 to 1.507) & 26 & 0.515 (0.249 to 0.780) & 20 & $0.007(-0.460$ to 0.474$)$ & 24 \\
\hline $16-19$ & 0.837 (0.465 to 1.209) & 8 & 0.643 (0.150 to 1.136) & 12 & 0.292 (0.048 to 0.535) & 10 & $-0.575(-1.212$ to 0.063$)$ & 14 \\
\hline $20-23$ & $-0.058(-0.322$ to 0.205$)$ & 5 & $-0.276(-0.541$ to -0.011$)$ & 5 & $-0.076(-0.339$ to 0.187$)$ & 5 & $0.154(-0.110$ to 0.417$)$ & 5 \\
\hline 24 & $0.096(-0.251$ to 0.443$)$ & 1 & $-0.014(-0.361$ to 0.333$)$ & 1 & $0.079(-0.268$ to 0.426$)$ & 1 & $0.005(-0.291$ to 0.402$)$ & 1 \\
\hline 36 & 1.492 (1.047 to 1.937) & 1 & 2.585 (2.049 to 3.121) & 1 & 1.402 (0.963 to 1.842) & 1 & $-0.993(-1.410$ to -0.577$)$ & 1 \\
\hline 48 & 0.575 (0.144 to 1.005) & 2 & 0.634 (0.202 to 1.066) & 2 & 1.164 (0.706 to 1.623) & 2 & $-1.020(-1.631$ to -0.409$)$ & 2 \\
\hline \multicolumn{9}{|l|}{ Intensity } \\
\hline Low & - & - & 1.191 (0.376 to 2.006) & 6 & 0.894 (0.076 to 1.711) & 3 & $-1.675(-3.175$ to -0.175$)$ & 6 \\
\hline Moderate & 0.505 (0.188 to 0.822) & 17 & $0.294(-0.159$ to 0.747$)$ & 20 & 0.506 (0.225 to 0.787) & 20 & $-0.023(-.0358$ to 0.313$)$ & 22 \\
\hline Vigorous & $0.124(-0.081$ to 0.330$)$ & 13 & 0.410 (0.171 to 0.649) & 22 & $0.176(-0.057$ to 0.409$)$ & 20 & $-0.285(-0.513$ to -0.057$)$ & 21 \\
\hline
\end{tabular}

Meta-ANOVA, meta-analysis of variance; TC, total cholesterol; TG, triglyceride; LDL, low-density lipoprotein; HDL, high-density lipoprotein; SMD, standardized mean difference; Cl, confidence interval; $n$, number of studies.

$\left(\mathrm{I}^{2}=65.3 \%\right)$ and $\%$ body fat $\left(\mathrm{I}^{2}=44.3 \%\right)$. The heterogeneity of $\%$ body fat was the only variable that was below moderate. These results indicate that the effects of exercise interventions on outward appearance, such as BMI and waist circumference, are larger than the effects on practical factors of obesity such as weight and \% body fat. Moreover, the effect of exercise on TG (SMD, 0.603) was larger than that on LDL (SMD, 0.406) and HDL (SMD, -0.222).

The results of the publication bias analysis were statistically significant for \% body fat, waist circumference, and HDL. In other words, the studies used in this meta-analysis adequately represent the population only for those three factors. With respect to the effects of the moderators on diet control, the SMDs for the cases with diet treatment were larger than those without diet control, as indicated by the weight, BMI, waist circumference, TC, and TG variables. Conversely, the SMDs with diet treatment were lower than those without diet control with respect to \% body fat, LDL, and HDL. In a meta-analysis by Vissers et al., ${ }^{75}$ supervised exerciseonly interventions had a greater effect than combined diet and exercise interventions. In a meta-analysis by Wu et al., ${ }^{76}$ a combined diet and exercise program provided greater long-term weight loss than a diet-only program. However, they concluded that both the diet-only and diet and exercise programs were associated with partial weight regain and that future studies should seek strategies to limit weight regain and achieve greater long-term weight loss.

In studies that considered the effects of moderators on exercise time per week, a medium or large SMD emerged for both body composition and lipid profile in programs that used more than 120 minutes of exercise per week. The ACSM's guidelines for exercise 


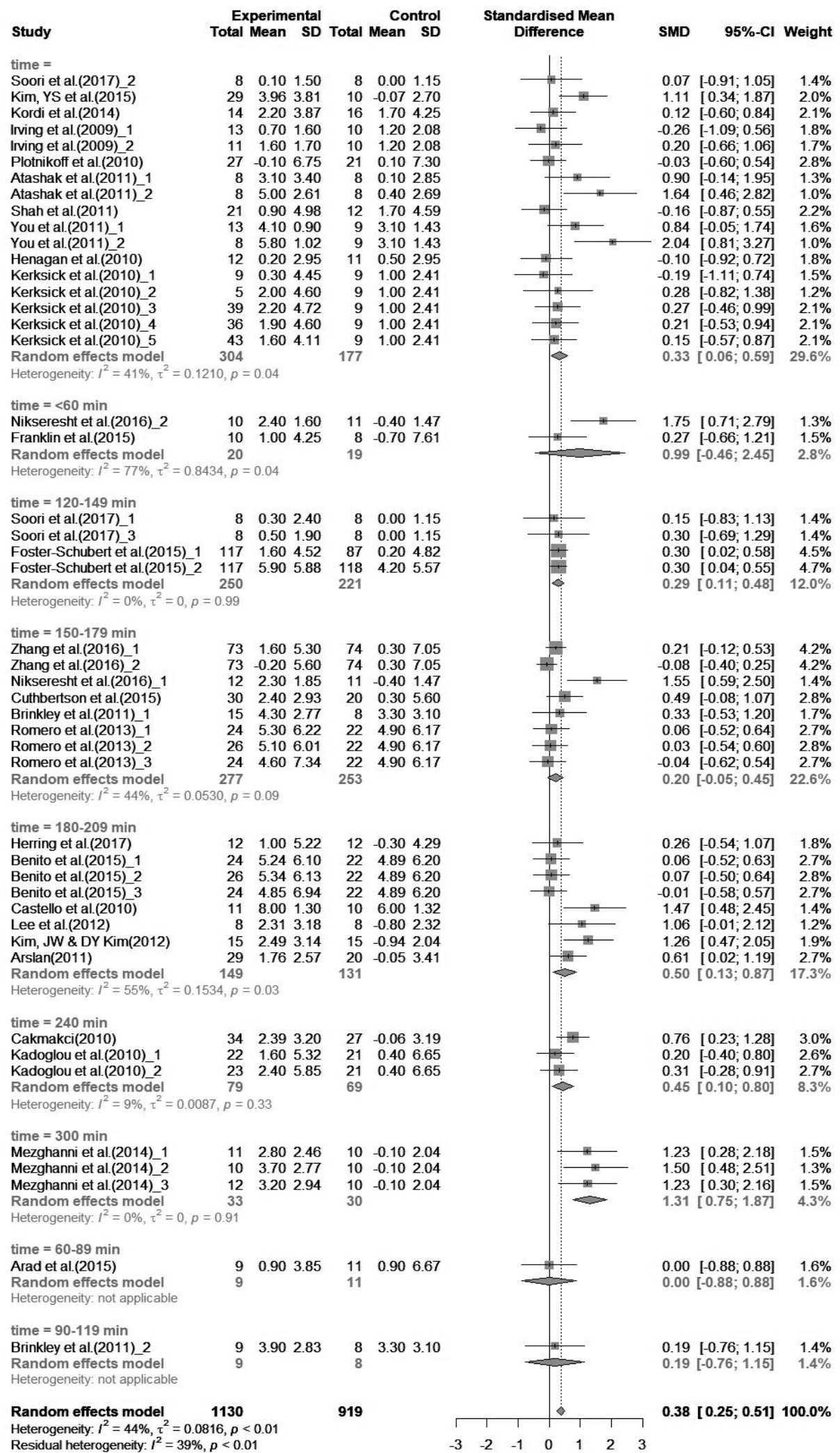

Figure 2. Forest plot of \% body fat by exercise time (minutes per week). The average standardized mean difference (SMD) for exercise time per week did not yield a specific pattern as the exercise time increased, possibly because of the small number of studies. "Total" in the figure indicates the number of subjects. Sixteen articles had several (2-5 pieces) comparisons, and each comparison was presented independently in the plot. SD, standard deviation; $\mathrm{Cl}$, confidence interval. 
testing and prescription recommend a minimum of $150 \mathrm{~min} \cdot \mathrm{wk}^{-1}$ $\left(30 \mathrm{~min} \cdot \mathrm{day}^{-1}\right)$ progressing to $300 \mathrm{~min} \cdot \mathrm{wk}^{-1}\left(60 \mathrm{~min} \cdot \mathrm{day}^{-1}\right)$ of moderate intensity exercise for overweight and obese individuals. ${ }^{73}$ Every study we considered here used a different combination of exercise intensity and duration.

An exercise duration of more than 8 weeks produced a medium or large SMD for body composition, as did a duration of 12 or more weeks for lipid profile. The ACSM's guidelines for exercise testing and prescription recommend a minimum reduction in initial body weight of 5\%-10\% over the course of 3-6 months for overweight and obese individuals. ${ }^{73}$ Furthermore, the "2018 Korean Society for the Study of Obesity Guideline for the Management of Obesity in Korea"74 states, "Physical activity is necessary for weight loss and maintenance. At the start, more than moderate physical activity is recommended. Moderate levels of physical activity include exercising between 30 minutes and 60 minutes five times per week. When including resistance exercise, it is recommended to engage in physical activity twice per week."

In this study, the average SMDs for weight loss by exercise duration were $0.237,0.455$, and 0.576 for $8-11$ weeks, $12-15$ weeks, and 16-19 weeks, respectively. In other words, the effect size was above medium after exercising for 16 weeks ( 4 months). Future studies should analyze both the rate of change in body weight and exercise duration together.

By exercise intensity, a medium or large SMD was observed in most cases with low and moderate intensity. Only waist circumference had an SMD above medium for vigorous intensity exercise. In a meta-analysis by Türk et al., ${ }^{3}$ training at high intensity was found to be superior to moderate exercise in reducing \% body fat in obese adults. That result is supported by some recent studies, but not by others. For example, a study by Keating et al. ${ }^{77}$ showed that high intensity interval training (HIIT) could improve fitness levels with only $50 \%-60 \%$ of the time commitment required by continuous aerobic exercise training (CONT). However, the CONT group showed a reduction in total body fat, whereas the HIIT group did not. In addition, Kemmler et al. ${ }^{78}$ found that HIIT provided more weight loss than moderate intensity continuous exercise(MICE) but produced no difference in body fat mass. Auriemma ${ }^{79} \mathrm{ex}^{-}$ plained that the available studies on HIIT in overweight and obese patients remain limited by their short duration, small number of participants, and variation in the intensity and duration of their "on" intervals. Researchers should also consider the risk of potential injuries, even though more vigorous exercises might provide additional benefits. $^{73}$

In conclusion, our novel finding is that the effect of exercise on obesity is larger in outward appearance (BMI, waist circumference) than in practical factors (weight, \% body fat). Moreover, the effect of exercise on TG was larger than that on LDL and HDL. With respect to exercise mode, the effects of exercise duration and intensity are more consistent and larger among obese adults than the effects of exercise time (minutes per week).

The limitations of this study include the generalization of the findings due to the small sample sizes in some of the studies, heterogeneity, and publication bias in the analysis of some variables. Some cases had very large SMDs with very small sample sizes. In future reviews, the number of studies included for each moderator effect analysis must be adequate to satisfy the normality of the statistical inference.

\section{CONFLICTS OF INTEREST}

The authors declare no conflict of interest.

\section{ACKNOWLEDGMENTS}

We thank the exercise committee members for participating in this article.

\section{AUTHOR CONTRIBUTIONS}

Study concept and design: KBK; acquisition of data: KK, CK, SJK, and HJK; analysis and interpretation of data: KBK and YAS; drafting of the manuscript: KBK and SY; critical revision of the manuscript: KBK and YAS; statistical analysis: KBK; administrative, technical, or material support: KBK and YAS; and study supervision: KK and YAS.

\section{SUPPLEMENTARY MATERIALS}

Supplementary Table 1. Classification of exercise intensity. 
Supplementary Figure 1. Flowchart for searching studies.

Supplementary Figure 2. Funnel plot of \%body fat.

They can be found via https://doi.org/10.7570/jomes.2019.28. 4.278 .

\section{REFERENCES}

1. Dietz WH. The effects of physical activity on obesity. Quest 2004;56:1-11.

2. Witham MD, Avenell A. Interventions to achieve long-term weight loss in obese older people: a systematic review and metaanalysis. Age Ageing 2010;39:176-84.

3. Türk Y, Theel W, Kasteleyn MJ, Franssen FM, Hiemstra PS, Rudolphus A, et al. High intensity training in obesity: a metaanalysis. Obes Sci Pract 2017;3:258-71.

4. Donner A, Klar N. The statistical analysis of kappa statistics in multiple samples. J Clin Epidemiol 1996;49:1053-8.

5. Moseley AM, Herbert RD, Sherrington C, Maher CG. Evidence for physiotherapy practice: a survey of the Physiotherapy Evidence Database (PEDro). Aust J Physiother 2002;48:43-9.

6. Fleiss JL. Statistical methods for rates and proportions. 2nd ed. New York (NY): John Wiley; 1981.

7. Scargle JD. Publication bias: the "file drawer" problem in scientific inference. J Sci Explor 2000;14:91-106.

8. Cohen J. Statistical power analysis for the behavioral sciences. 2nd ed. Hillsdale (NJ): Lawrence Erlbaum Associates; 1988.

9. Herring LY, Stevinson C, Carter P, Biddle SJ, Bowrey D, Sutton C, et al. The effects of supervised exercise training 12-24 months after bariatric surgery on physical function and body composition: a randomised controlled trial. Int J Obes (Lond) 2017;41:909-16.

10. Marcon ER, Baglioni S, Bittencourt L, Lopes CL, Neumann $\mathrm{CR}$, Trindade MR. What is the best treatment before bariatric surgery? Exercise, exercise and group therapy, or conventional waiting: a randomized controlled trial. Obes Surg 2017;27: 763-73.

11.Zhang HJ, Pan LL, Ma ZM, Chen Z, Huang ZF, Sun Q, et al. Long-term effect of exercise on improving fatty liver and cardiovascular risk factors in obese adults: a 1-year follow-up study. Diabetes Obes Metab 2017;19:284-9.
12. Ash GI, Taylor BA, Thompson PD, MacDonald HV, Lamberti $\mathrm{L}, \mathrm{Chen} \mathrm{MH}$, et al. The antihypertensive effects of aerobic versus isometric handgrip resistance exercise. J Hypertens 2017; 35:291-9.

13. Freitas PD, Ferreira PG, Silva AG, Stelmach R, Carvalho-Pinto RM, Fernandes FL, et al. The role of exercise in a weight-loss program on clinical control in obese adults with asthma: a randomized controlled trial. Am J Respir Crit Care Med 2017; 195:32-42.

14. Baillot A, Mampuya WM, Dionne IJ, Comeau E, Méziat-Burdin A, Langlois MF. Impacts of supervised exercise training in addition to interdisciplinary lifestyle management in subjects awaiting bariatric surgery: a randomized controlled study. Obes Surg 2016;26:2602-10.

15. Nikseresht M, Hafezi Ahmadi MR, Hedayati M. Detraininginduced alterations in adipokines and cardiometabolic risk factors after nonlinear periodized resistance and aerobic interval training in obese men. Appl Physiol Nutr Metab 2016;41: 1018-25.

16. Nunes PR, Barcelos LC, Oliveira AA, Furlanetto Júnior R, Martins FM, Orsatti CL, et al. Effect of resistance training on muscular strength and indicators of abdominal adiposity, metabolic risk, and inflammation in postmenopausal women: controlled and randomized clinical trial of efficacy of training volume. Age (Dordr) 2016;38:40.

17. Soori R, Rezaeian N, Khosravi N, Ahmadizad S, Taleghani HM, Jourkesh M, et al. Effects of water-based endurance training, resistance training, and combined water and resistance training programs on visfatin and ICAM-1 levels in sedentary obese women. Sci Sport 2017;32:144-51.

18. Cuthbertson DJ, Shojaee-Moradie F, Sprung VS, Jones H, Pugh CJ, Richardson P, et al. Dissociation between exercise-induced reduction in liver fat and changes in hepatic and peripheral glucose homoeostasis in obese patients with non-alcoholic fatty liver disease. Clin Sci (Lond) 2016;130:93-104.

19. Rafraf M, Karimi M, Jafari A. Effect of L-carnitine supplementation in comparison with moderate aerobic training on serum inflammatory parameters in healthy obese women. J Sports Med Phys Fitness 2015;55:1363-70.

20. Abdelaal AA, Mohamad MA. Obesity indices and haemody- 
namic response to exercise in obese diabetic hypertensive patients: randomized controlled trial. Obes Res Clin Pract 2015; 9:475-86.

21. Arad AD, DiMenna FJ, Thomas N, Tamis-Holland J, Weil R, Geliebter A, et al. High-intensity interval training without weight loss improves exercise but not basal or insulin-induced metabolism in overweight/obese African American women. J Appl Physiol (1985) 2015;119:352-62.

22. Kim YS, Nam JS, Yeo DW, Kim KR, Suh SH, Ahn CW. The effects of aerobic exercise training on serum osteocalcin, adipocytokines and insulin resistance on obese young males. Clin Endocrinol (Oxf) 2015;82:686-94.

23. Benito PJ, Bermejo LM, Peinado AB, López-Plaza B, Cupeiro $\mathrm{R}$, Szendrei B, et al. Change in weight and body composition in obese subjects following a hypocaloric diet plus different training programs or physical activity recommendations. J Appl Physiol (1985) 2015;118:1006-13.

24. Kordi R, Dehghani S, Noormohammadpour P, Rostami M, Mansournia MA. Effect of abdominal resistance exercise on abdominal subcutaneous fat of obese women: a randomized controlled trial using ultrasound imaging assessments. J Manipulative Physiol Ther 2015;38:203-9.

25. Park SM, Kwak YS, Ji JG. The effects of combined exercise on health-related fitness, endotoxin, and immune function of postmenopausal women with abdominal obesity. J Immunol Res 2015;2015:830567.

26. Ross R, Hudson R, Stotz PJ, Lam M. Effects of exercise amount and intensity on abdominal obesity and glucose tolerance in obese adults: a randomized trial. Ann Intern Med 2015;162: 325-34.

27. Pugh CJ, Spring VS, Kemp GJ, Richardson P, Shojaee-Moradie F, Umpleby AM, et al. Exercise training reverses endothelial dysfunction in nonalcoholic fatty liver disease. Am J Physiol Heart Circ Physiol 2014;307:H1298-306.

28. Nikseresht M, Agha-Alinejad H, Azarbayjani MA, Ebrahim K. Effects of nonlinear resistance and aerobic interval training on cytokines and insulin resistance in sedentary men who are obese. J Strength Cond Res 2014;28:2560-8.

29. Herring LY, Wagstaff C, Scott A. The efficacy of 12 weeks supervised exercise in obesity management. Clin Obes 2014;4:
220-7.

30. Irving BA, Weltman JY, Patrie JT, Davis CK, Brock DW, Swift D, et al. Effects of exercise training intensity on nocturnal growth hormone secretion in obese adults with the metabolic syndrome. J Clin Endocrinol Metab 2009;94:1979-86.

31. Straznicky NE, Lambert EA, Nestel PJ, McGrane MT, Dawood T, Schlaich MP, et al. Sympathetic neural adaptation to hypocaloric diet with or without exercise training in obese metabolic syndrome subjects. Diabetes 2010;59:71-9.

32. Ibáñez J, Izquierdo M, Martínez-Labari C, Ortega F, Grijalba A, Forga L, et al. Resistance training improves cardiovascular risk factors in obese women despite a significative decrease in serum adiponectin levels. Obesity (Silver Spring) 2010;18:535-41.

33. Christiansen T, Paulsen SK, Bruun JM, Pedersen SB, Richelsen B. Exercise training versus diet-induced weight-loss on metabolic risk factors and inflammatory markers in obese subjects: a 12-week randomized intervention study. Am J Physiol Endocrinol Metab 2010;298:E824-31.

34. Sartor F, de Morree HM, Matschke V, Marcora SM, Milousis A, Thom JM, et al. High-intensity exercise and carbohydrate-reduced energy-restricted diet in obese individuals. Eur J Appl Physiol 2010;110:893-903.

35. Plotnikoff RC, Eves N, Jung M, Sigal RJ, Padwal R, Karunamuni N. Multicomponent, home-based resistance training for obese adults with type 2 diabetes: a randomized controlled trial. Int J Obes (Lond) 2010;34:1733-41.

36. Yamaguchi T, Saiki A, Endo K, Miyashita Y, Shirai K. Effect of exercise performed at anaerobic threshold on serum growth hormone and body fat distribution in obese patients with type 2 diabetes. Obes Res Clin Pract 2011;5:e1-78.

37. Straznicky NE, Grima MT, Lambert EA, Eikelis N, Dawood T, Lambert GW, et al. Exercise augments weight loss induced improvement in renal function in obese metabolic syndrome individuals. J Hypertens 2011;29:553-64.

38. Lim SS, Norman RJ, Clifton PM, Noakes M. The effect of comprehensive lifestyle intervention or metformin on obesity in young women. Nutr Metab Cardiovasc Dis 2011;21:261-8.

39. Brinkley TE, Ding J, Carr JJ, Nicklas BJ. Pericardial fat loss in postmenopausal women under conditions of equal energy deficit. Med Sci Sports Exerc 2011;43:808-14. 
40. Brinkley TE, Wang X, Kume N, Mitsuoka H, Nicklas BJ. Caloric restriction, aerobic exercise training and soluble lectin-like oxidized LDL receptor-1 levels in overweight and obese postmenopausal women. Int J Obes (Lond) 2011;35:793-9.

41. Atashak S, Peeri M, Jafari A, Ali Azarbayjani A. Effects of ginger supplementation and resistance training on lipid profiles and body composition in obese men. J Med Plants Res 2011; 5:3827-32.

42. Shah M, Snell PG, Rao S, Adams-Huet B, Quittner C, Livingston $\mathrm{EH}$, et al. High-volume exercise program in obese bariatric surgery patients: a randomized, controlled trial. Obesity (Silver Spring) 2011;19:1826-34.

43. Castello V, Simões RP, Bassi D, Catai AM, Arena R, BorghiSilva A. Impact of aerobic exercise training on heart rate variability and functional capacity in obese women after gastric bypass surgery. Obes Surg 2011;21:1739-49.

44. You T, Disanzo BL, Wang X, Yang R, Gong D. Adipose tissue endocannabinoid system gene expression: depot differences and effects of diet and exercise. Lipids Health Dis 2011;10:194.

45. Henagan TM, Phillips MD, Cheek DJ, Kirk KM, Barbee JJ, Stewart LK. The melanocortin 3 receptor: a novel mediator of exercise-induced inflammation reduction in postmenopausal women? J Aging Res 2011;2011:512593.

46. Lee JA, Kim JW, Kim DY. Effects of yoga exercise on serum adiponectin and metabolic syndrome factors in obese postmenopausal women. Menopause 2012;19:296-301.

47. Sullivan S, Kirk EP, Mittendorfer B, Patterson BW, Klein S. Randomized trial of exercise effect on intrahepatic triglyceride content and lipid kinetics in nonalcoholic fatty liver disease. Hepatology 2012;55:1738-45.

48. Swift DL, Earnest CP, Blair SN, Church TS. The effect of different doses of aerobic exercise training on endothelial function in postmenopausal women with elevated blood pressure: results from the DREW study. Br J Sports Med 2012;46:753-8.

49. Foster-Schubert KE, Alfano CM, Duggan CR, Xiao L, Campbell KL, Kong A, et al. Effect of diet and exercise, alone or combined, on weight and body composition in overweight-to-obese postmenopausal women. Obesity (Silver Spring) 2012;20: 1628-38.

50. Abd El-Kader SM, Al-Jiffri OH, Al-Shreef FM. Aerobic exer- cises alleviate symptoms of fatigue related to inflammatory cytokines in obese patients with type 2 diabetes. Afr Health Sci 2015;15:1142-8.

51. Wong A, Alvarez-Alvarado S, Jaime SJ, Kinsey AW, Spicer MT, Madzima TA, et al. Combined whole-body vibration training and l-citrulline supplementation improves pressure wave reflection in obese postmenopausal women. Appl Physiol Nutr Metab 2016;41:292-7.

52. Osama AJ, Shehab Ael-K. Psychological wellbeing and biochemical modulation in response to weight loss in obese type 2 diabetes patients. Afr Health Sci 2015;15:503-12.

53. Franklin NC, Robinson AT, Bian JT, Ali MM, Norkeviciute E, McGinty P, et al. Circuit resistance training attenuates acute exertion-induced reductions in arterial function but not inflammation in obese women. Metab Syndr Relat Disord 2015;13: 227-34.

54. Coen PM, Tanner CJ, Helbling NL, Dubis GS, Hames KC, Xie $\mathrm{H}$, et al. Clinical trial demonstrates exercise following bariatric surgery improves insulin sensitivity. J Clin Invest 2015; 125:248-57.

55. Romero Moraleda B, Morencos E, Peinado AB, Bermejo L, Gómez Candela C, Benito PJ, et al. Can the exercise mode determine lipid profile improvements in obese patients? Nutr Hosp 2013;28:607-17.

56. Figueroa A, Arjmandi BH, Wong A, Sanchez-Gonzalez MA, Simonavice E, Daggy B. Effects of hypocaloric diet, low-intensity resistance exercise with slow movement, or both on aortic hemodynamics and muscle mass in obese postmenopausal women. Menopause 2013;20:967-72.

57. Bhutani S, Klempel MC, Kroeger CM, Trepanowski JF, Varady KA. Alternate day fasting and endurance exercise combine to reduce body weight and favorably alter plasma lipids in obese humans. Obesity (Silver Spring) 2013;21:1370-9.

58. Figueroa A, Vicil F, Sanchez-Gonzalez MA, Wong A, Ormsbee MJ, Hooshmand S, et al. Effects of diet and/or low-intensity resistance exercise training on arterial stiffness, adiposity, and lean mass in obese postmenopausal women. Am J Hypertens 2013;26:416-23.

59. Trussardi Fayh AP, Lopes AL, Fernandes PR, Reischak-Oliveira A, Friedman R. Impact of weight loss with or without exercise 
on abdominal fat and insulin resistance in obese individuals: a randomised clinical trial. Br J Nutr 2013;110:486-92.

60. García-Unciti M, Izquierdo M, Idoate F, Gorostiaga E, Grijalba A, Ortega-Delgado F, et al. Weight-loss diet alone or combined with progressive resistance training induces changes in association between the cardiometabolic risk profile and abdominal fat depots. Ann Nutr Metab 2012;61:296-304.

61. Tseng ML, Ho CC, Chen SC, Huang YC, Lai CH, Liaw YP. A simple method for increasing levels of high-density lipoprotein cholesterol: a pilot study of combination aerobic- and resistanceexercise training. Int J Sport Nutr Exerc Metab 2013;23:271-81.

62. Fayh AP, Lopes AL, da Silva AM, Reischak-Oliveira A, Friedman R. Effects of $5 \%$ weight loss through diet or diet plus exercise on cardiovascular parameters of obese: a randomized clinical trial. Eur J Nutr 2013;52:1443-50.

63. Kim JW, Kim DY. Effects of aerobic exercise training on serum sex hormone binding globulin, body fat index, and metabolic syndrome factors in obese postmenopausal women. Metab Syndr Relat Disord 2012;10:452-7.

64. Snel M, Gastaldelli A, Ouwens DM, Hesselink MK, Schaart G, Buzzigoli E, et al. Effects of adding exercise to a 16-week very low-calorie diet in obese, insulin-dependent type 2 diabetes mellitus patients. J Clin Endocrinol Metab 2012;97:2512-20.

65. Cakmakçi O. The effect of 8 week pilates exercise on body composition in obese women. Coll Antropol 2011;35:1045-50.

66. Masuo K, Rakugi H, Ogihara T, Lambert GW. Different mechanisms in weight loss-induced blood pressure reduction between a calorie-restricted diet and exercise. Hypertens Res 2012;35: 41-7.

67. Kerksick CM, Wismann-Bunn J, Fogt D, Thomas AR, Taylor L, Campbell BI, et al. Changes in weight loss, body composition and cardiovascular disease risk after altering macronutrient distributions during a regular exercise program in obese women. Nutr J 2010;9:59.

68. Kadoglou NP, Iliadis F, Sailer N, Athanasiadou Z, Vitta I, Kapelouzou A, et al. Exercise training ameliorates the effects of rosiglitazone on traditional and novel cardiovascular risk factors in patients with type 2 diabetes mellitus. Metabolism 2010;59: 599-607.
69. Murakami T, Horigome H, Tanaka K, Nakata Y, Katayama Y, Matsui A. Effects of diet with or without exercise on leptin and anticoagulation proteins levels in obesity. Blood Coagul Fibrinolysis 2007;18:389-94.

70. Arslan F. The effects of an eight-week step-aerobic dance exercise programme on body composition parameters in middleaged sedentary obese women. Int Sport Med J 2011;12:160-8.

71. Mezghanni N, Lahyani A, Jamoussi K, Mnif M, Masmoudi L, Abid M, et al. Effect of exercise intensity on body composition and cardiovascular disease risk factors in sedentary young obese women. Int Sport Med J 2014;15:415-24.

72. Rshikesan PB, Subramanya P. Effect of integrated approach of yoga therapy on male obesity and psychological parameters: a randomised controlled trial. J Clin Diagn Res 2016;10:KC01-6.

73. American College of Sports Medicine. ACSM's guidelines for exercise testing and prescription. 9th ed. Philadelphia (PA): Lippincott Williams \& Wilkins; 2014.

74. Seo MH, Lee WY, Kim SS, Kang JH, Kang JH, Kim KK, et al. 2018 Korean Society for the Study of Obesity Guideline for the management of obesity in Korea. J Obes Metab Syndr 2019;28:40-5.

75. Vissers D, Hens W, Hansen D, Taeymans J. The effect of diet or exercise on visceral adipose tissue in overweight youth. Med Sci Sports Exerc 2016;48:1415-24.

76. Wu T, Gao X, Chen M, van Dam RM. Long-term effectiveness of diet-plus-exercise interventions vs. diet-only interventions for weight loss: a meta-analysis. Obesity Reviews 2009; 10:313-23.

77. Keating SE, Machan EA, O'Connor HT, Gerofi JA, Sainsbury A, Caterson ID, et al. Continuous exercise but not high intensity interval training improves fat distribution in overweight adults. J Obes 2014;2014:834865.

78. Kemmler W, Scharf M, Lell M, Petrasek C, von Stengel S. High versus moderate intensity running exercise to impact cardiometabolic risk factors: the randomized controlled RUSH-study. Biomed Res Int 2014;2014:843095.

79. Auriemma A. High-intensity interval training versus traditional exercise in adults with overweight and obesity. Bariatric Times 2017;14:12-5. 